\title{
Fluid Antenna Multiple Access
}

\author{
Kai-Kit Wong, Fellow, IEEE, and Kin-Fai Tong, Senior Member, IEEE
}

\begin{abstract}
Fluid antenna system represents an emerging technology that enables an antenna to switch its physical location in a predefined space. This paper explores the potential of using a single fluid antenna at each mobile user for multiple access, which we refer to it as fluid antenna multiple access (FAMA). FAMA exploits spatial moments of deep fade suffered by the interference to achieve a favourable channel condition for the desired signal, without requiring sophisticated signal processing. We analyze the FAMA network by first deriving the outage probability of the signal-to-interference ratio (SIR) in a double integral form. We then obtain an outage probability upper bound in closed form and an average outage rate lower bound for the FAMA system, with an arbitrary number of interferers, from which the multiplexing gain of FAMA is characterized. We also estimate how large the number of locations is required to achieve a given multiplexing gain using fluid antennas with a given size. Results show that it is possible for FAMA to support hundreds of users using only one fluid antenna of a few wavelengths of space at each user, giving rise to significant gain in the average network outage rate.
\end{abstract}

Index Terms-FAMA, Fluid antennas, MIMO, Multiple access, Multiplexing gain, Selection combining, Outage.

\section{INTRODUCTION}

Over the past twenty years, multiple-input multiple-output (MIMO) has revolutionized mobile communications by creating bandwidth from space, independent of frequency and time resources. The enormous diversity and multiplexing gains inherent in spatially uncorrelated channels at multiple antennas have proven to deliver extraordinary performance for pointto-point communication links, e.g., [1]-[5]. Multiuser MIMO, nevertheless, is arguably an even greater contribution, since it multiplexes users entirely in the spatial domain and upscales the network capacity with the number of antennas at the base stations (BSs) and/or mobile stations (MSs), e.g., [6]-[9].

Multiuser MIMO focuses predominantly on the signal processing and coding at the BS side, leaving the MSs a relatively easier task for communications. This makes sense because BSs tend to have much higher processing capability but it also means that the BSs could quickly become overwhelmed by the processing requirements of a huge number of users. Although massive MIMO in 5G greatly simplifies the signal processing of multiuser signals by the virtue of the law of large numbers by adopting a massive number of antennas at the BS [8], [9], the number of antennas used needs to be much greater than the number of supported users. Also, for $5 \mathrm{G}$ as an example, only 64 antennas are employed and channel inverses are required to eliminate the inter-user interference [10]-[12], let alone other associated processing tasks such as pilot decontamination [13], power control [14], user-cell association [15], and etc. The

The work is supported in part by EPSRC under grant EP/T015985/1.

K. K. Wong and K. F. Tong are with the Department of Electronic and Electrical Engineering, University College London, London WC1E 7JE, UK. fact that the latest trends require resource management to be content-centric [16], [17], further puts strains on the BSs.

\section{Can space-division multiple-access be much simpler, at least conceptually?}

To answer this question, we begin by reviewing the fading phenomenon. Multipath fading, also referred to as short-term fading, is a result of the superposition of radio waves travelling via different paths due to scattering in the environment from a transmitter to a receiver. The complex interaction between the paths of the physical environment gives rise to a mixture of construction or destruction of multipath signals, resulting in a random signal envelope at the receiver. In a deep fade, as deep as $50 \mathrm{~dB}$ drop in the field strength is not uncommon. A great deal of research has been carried out to avoid these deep fades through smart resource management and advanced coding to provide some stability in communications.

Hypothetically, if we can identify and access the deep fades of interference, then it will be possible to utilize them to our advantage and exploit those moments of deep fade of interference for multiple access. In other words, the interference will disappear naturally if it is in a deep fade, providing a window of opportunity for interference-free communications. In order to achieve this, it will require a technology that allows us to skim through a collection of fading envelopes and exploit their ups and downs. The technology that makes this realizable is fluid antenna which refers to any software-controllable fluidic, conductive radiating structure that can alter their shape and/or position to reconfigure the polarization, operating frequency, radiation pattern and etc., and it may include designs involving no fluidic materials if they can mimic the agility [18].

The term 'fluid antenna' was first introduced by Kar et al. in [19] where conductive fluids as materials for an antenna were investigated. However, using fluidic structures for an antenna can be traced back to even earlier [20] when mercury was used to make a circular patch liquid microstrip antenna. Afterwards, reconfigurable fluid antennas also began to emerge, resulting in a variety of reconfigurable functionalities [21]-[28]. Some were based on liquid metal antennas [21]-[23] whereas others used more accessible ionized solutions such as sodium chloride and potassium chloride for antennas [24]-[28]. Also, there is the approach of using pixel electronic switches in designing reconfigurable antennas and such idea could be an alternative version of 'fluid' antenna [29]. Furthermore, there were high profile successes in using seawater for antennas. The first was conducted by Mitsubishi Electric which illustrated a radiation efficiency of $70 \%$ [30]. Remarkably, Xing et al. even devised an advanced saltwater-based antenna to achieve 360-degree beam-steering between 334 and $488 \mathrm{MHz}$ [31], [32]. A more 
thorough, contemporary survey on this topic can be found in [33], [34]. Evidently, fluid antennas are on their way to impact future-generation mobile communications [35].

This paper uses the term 'fluid antenna' to represent a reconfigurable system that can automatically switch the antenna position to the best of $N$ fixed locations (referred to as 'ports') within a given space instantly. The diversity benefit of fluid antenna for single-user communication was analyzed in terms of outage probability in [36] while [37] has already examined the ergodic capacity of a single-user fluid antenna system. It was revealed that with a space of half a wavelength or less, a single fluid antenna system with only one RF chain can obtain as much capacity as a maximum ratio combining system with many spatially uncorrelated antennas. Different from [36] and [37] which addressed single-user systems, this paper proposes to utilize fluid antennas for multiuser communications for an interference channel in which there are multiple uncoordinated pairs of transmitters and receivers in flat-fading scenarios.

We explain this concept using Fig. 1 which shows a possible sitation with two transmitter-receiver pairs and both MSs (i.e., users) deploy a fluid antenna system for reception. The fluid antenna system for user 1 has a space of $W_{1} \lambda$ with $\lambda$ denoting the wavelength and can observe the fading envelopes in the available space and switch the antenna to the position where the signal for user 2 is in a deep fade. The same happens for user 2 to mitigate the interference from BS transmitter 1. As a consequence, the inter-user interference is naturally avoided by picking the right moment across the space in the manner of selection combining ${ }^{1}$ where the signal-to-interference ratio (SIR) is maximized for multiple access. We coin this scheme as fluid antenna multiple access (FAMA) where each MS (or a FAMA user) has a single RF-chain $N$-port fluid antenna to always switch to the position with the strongest SIR.

FAMA is entirely user-centric and requires no coordination between transmitters and receivers, and needs no prior channel knowledge of the interfering links. Furthermore, FAMA users are completely decentralized and the processing on one user has no impact on other users. The interference suppression capability comes naturally from the fading phenomenon, and the degree of freedom is dictated by the richness of scattering in the environment and the system ability to exploit the fading envelopes through the parameters such as the number of ports, $N$, and the size of the fluid antenna, $W \lambda$, at each user.

The objective of this paper is to characterize the achievable performance of FAMA and understand how the performance scales with the number of ports, $N$, and the size of the fluid antenna at each user, $W \lambda$. To this end, we derive the outage probability for the SIR which reveals the interference suppression capability at the user level. The exact outage probability for the SIR is obtained in a double integral form. Then we

\footnotetext{
${ }^{1}$ Although the signal processing for a fluid antenna resembles selection combining in traditional multi-antenna systems, it should be pointed out that selection combining has never been proposed for multiple access because it would have required too many antennas to be useful and the $\frac{\lambda}{2}$-rule between adjacent antennas would prove the idea to be infeasible. A key novelty of this paper therefore lies in our result that multiple access is achieved by closely located, spatially correlated ports in the tight space of a fluid antenna. This takes advantage of the advances in fluid-like antenna technologies, making possible a large number of switchable ports in a small space.
}

propose an outage probability upper bound in closed form to unpack the relationship between the operational parameters and the achievable performance. Subsequently, a lower bound for the average outage rate in closed form is presented, which enables our multiplexing gain analysis of the FAMA network. Furthermore, we obtain a sufficient condition on how large $N$ would be required for the fluid antenna at each FAMA user in order to achieve a certain multiplexing gain.

It is worth emphasizing that the above-mentioned analysis was not required in [36], [37] in which the aim was to understand the diversity gain and how much it could be translated into the signal-to-noise ratio (SNR) based outage probability performance and ergodic capacity. The interference immunity analysis via the SIR-based outage probability and the capacity scaling of FAMA against different system parameters as well as fluid antenna size analysis with respect to capacity scaling, are all new in this paper. In addition to the above contributions, we summarize some of our key findings as follows:

- The size of fluid antenna at the MS is important but has a diminishing return after $\frac{\lambda}{2}$, and its impact on the network outage rate is insignificant if the size grows beyond $\frac{\lambda}{2}{ }^{2}$

- If the number of ports is sufficiently large, a single fluid antenna at the MS can achieve any arbitrarily small SIR outage probability, suggesting the feasibility of FAMA.

- The network outage rate scales linearly with the number of ports at each FAMA user but is ultimately limited by the number of coexisting users (or MSs).

- The multiplexing gain scales linearly with the number of ports, and is inversely proportional to the SIR target but is again upper bounded by the number of coexisting MSs.

The rest of the paper is organized as follows. In Section II, we introduce the network model of FAMA under the setting of an interference channel. Our main results will be presented in Section III. Section IV attempts to make some interesting observations of FAMA by presenting some numerical results. Finally, we provide some concluding remarks in Section V.

\section{NETWORK MODEL}

\section{A. Single-User Fluid Antenna System}

Before we consider a network of fluid antenna system users, we introduce the model for a single MS that is equipped with a fluid antenna. The MS operates as a receiver and has a 'fluid' antenna whose location can be switched instantly to one of the $N$ preset locations evenly distributed along a linear dimension of length, $W \lambda$. We refer to a switchable location as 'port' and all the ports share a common RF chain. Any delay for switching between the ports is assumed negligible, and ignored in this paper. Moreover, in order to make possible performance analysis using communication theory, we use an abstraction to model the concept and treat each port as an ideal point antenna.

The first port is the reference location, from which the displacement of the $k$-th port is measured:

$$
d_{k}=\left(\frac{k-1}{N-1}\right) W \lambda, \text { for } k=1,2, \ldots, N .
$$

\footnotetext{
${ }^{2}$ That being said, space can be extremely important as $N$ is anticipated to be constrained by the space available for implementation.
} 


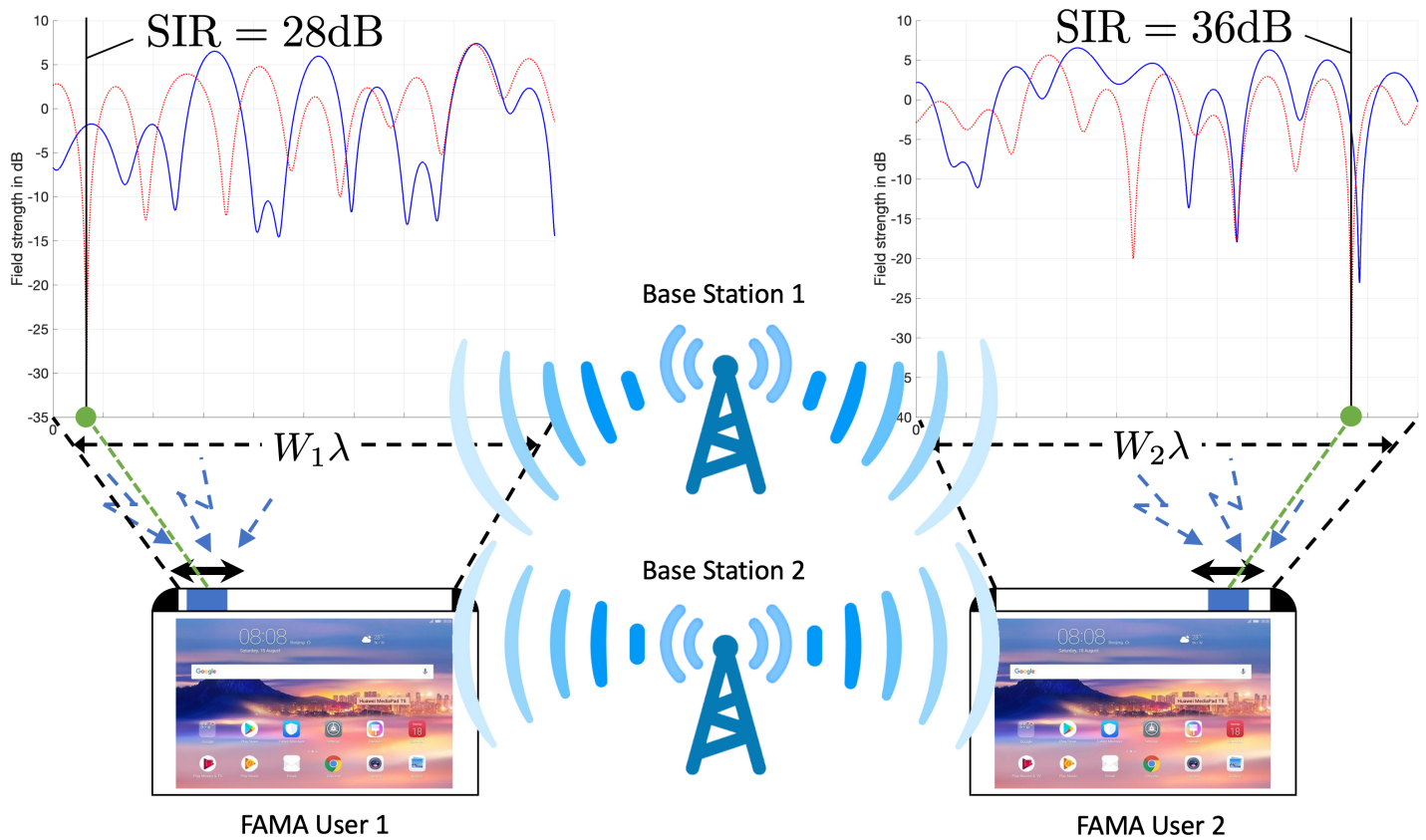

Fig. 1. The concept of FAMA where each FAMA user is able to switch its antenna to the position in which the interference is in a deep fade for maximizing the SIR. FAMA works for interference channels as the channels for the desired signal and interference have different fading envelopes.

The received signal at the $k$-th port is modelled as

$$
z_{k}=g_{k} s+\eta_{k}
$$

where the time index is omitted, $g_{k}$ is the complex channel envelope experienced by the $k$-th port, which follows a circularly symmetric complex Gaussian distribution with zero mean and variance of $\sigma^{2}, \eta_{k}$ is the complex additive white Gaussian noise (AWGN) at the $k$-th port with zero mean and variance of $\sigma_{\eta}^{2}$, and $s$ denotes the transmitted data symbol. In our model, the amplitude of the channel, $\left|g_{k}\right|$, is assumed Rayleigh distributed, with the probability density function (pdf)

$$
p_{\left|g_{k}\right|}(r)=\frac{2 r}{\sigma^{2}} e^{-\frac{r^{2}}{\sigma^{2}}}, \text { for } r \geq 0 \text { with } \mathrm{E}\left[\left|g_{k}\right|^{2}\right]=\sigma^{2} \text {. }
$$

The average received SNR at each port is given by

$$
\Gamma=\sigma^{2} \frac{\mathrm{E}\left[|s|^{2}\right]}{\sigma_{\eta}^{2}} \equiv \sigma^{2} \Theta, \text { where } \Theta \triangleq \frac{\mathrm{E}\left[|s|^{2}\right]}{\sigma_{\eta}^{2}}
$$

The channels $\left\{g_{k}\right\}_{\forall n}$ are considered to be correlated as they can be arbitrarily close to each other.

With 2-D isotropic scattering and an isotropic receiver port, it is known that the cross-correlation functions of the channel satisfy [38]

$$
\phi_{g_{k} g_{\ell}}\left(d_{k}-d_{\ell}\right)=\frac{\sigma^{2}}{2} J_{0}\left(\frac{2 \pi(k-\ell)}{N-1} W\right) \text {, }
$$

where $J_{0}(\cdot)$ denotes the zero-order Bessel function of the first kind. For ease of exposition, we find it useful to parameterize the channels at the $N$ antenna ports by

$$
\left\{\begin{aligned}
g_{1} & =\sigma x_{0}+j \sigma y_{0} \\
g_{k} & =\sigma\left(\sqrt{1-\mu_{k}^{2}} x_{k}+\mu_{k} x_{0}\right) \\
& +j \sigma\left(\sqrt{1-\mu_{k}^{2}} y_{k}+\mu_{k} y_{0}\right), \text { for } k=2, \ldots, N,
\end{aligned}\right.
$$

where $x_{0}, \ldots, x_{N}, y_{0}, \ldots, y_{N}$ are all independent Gaussian random variables with zero mean and variance of $\frac{1}{2}$, and $\left\{\mu_{k}\right\}$ are the cross-correlation parameters that can be chosen appropriately to specify the correlation among $\left\{g_{k}\right\}$. Based on this model, $\mathrm{E}\left[\left|g_{k}\right|^{2}\right]=\sigma^{2} \forall k$ and due to (5), we have

$$
\mu_{k}=J_{0}\left(\frac{2 \pi(k-1)}{N-1} W\right), \text { for } k=2, \ldots, N .
$$

In this single-user model, the instantaneous SNR at the $k$-th port is given by $\left|g_{k}\right|^{2} \Theta$ and the achievable SNR for the fluid antenna is hence $\Theta \max _{k}\left\{\left|g_{k}\right|^{2}\right\}$. An important performance metric is the probability of an outage event

$$
\mathcal{O}_{\mathrm{SNR}}=\left\{\max _{k}\left\{\left|g_{k}\right|^{2}\right\}<\frac{\bar{\gamma}}{\Theta} \equiv \gamma\right\},
$$

where $\bar{\gamma}$ is the target SNR and $\gamma$ is the normalized target SNR. The outage probability, $\operatorname{Prob}\left(\mathcal{O}_{\mathrm{SNR}}\right)$, has been studied in [36].

\section{B. FAMA}

In this paper, we consider an interference channel with $N_{\mathrm{I}}+$ 1 pairs of transmitter and receiver. ${ }^{3}$ In what follows, each user

${ }^{3}$ While this paper considers an interference channel, the principle of FAMA works equally well in downlink and uplink channels. In fact, the models for downlink and uplink channels will be the same as the interference channel model if for downlink channels, each user's signal comes from a different antenna at the BS while for uplink channels, the BS should have a dedicated fluid antenna for receiving each user's signal. 
is affected by $N_{\mathrm{I}}$ interferers. Therefore, (2) becomes

$$
z_{k}=g_{k} s+\sum_{i=1}^{N_{\mathrm{I}}} g_{k}^{(i)} s_{i}+\eta_{k} \equiv g_{k} s+g_{k}^{\mathrm{I}}+\eta_{k},
$$

in which $s_{i}$ is the transmitted data from the $i$-th interferer and $g_{k}^{(i)}$ denotes its corresponding channel. The parameters for the interferers are modelled in the same way as the desired signal. Since $\left\{g_{k}^{(i)}\right\}_{\forall i}$ are all complex Gaussian, we can model the total interference at the $k$-th port as $g_{k}^{\mathrm{I}}=\sum_{i=1}^{N_{\mathrm{I}}} g_{k}^{(i)} s_{i}$ which is again complex Gaussian distributed with zero mean and variance of some $\sigma_{\mathrm{I}}^{2}=\sum_{i=1}^{N_{\mathrm{I}}} \mathrm{E}\left[\left|g_{k}^{(i)}\right|^{2}\right] \mathrm{E}\left[\left|s_{i}\right|^{2}\right]$.

Following (6), we can also model $\left\{g_{k}^{\mathrm{I}}\right\}$ by

$$
\left\{\begin{aligned}
g_{1}^{\mathrm{I}} & =\sigma_{\mathrm{I}} x_{0}^{\mathrm{I}}+j \sigma_{\mathrm{I}} y_{0}^{\mathrm{I}} \\
g_{k}^{\mathrm{I}} & =\sigma_{\mathrm{I}}\left(\sqrt{1-\mu_{k}^{2}} x_{k}^{\mathrm{I}}+\mu_{k} x_{0}^{\mathrm{I}}\right) \\
& +j \sigma_{\mathrm{I}}\left(\sqrt{1-\mu_{k}^{2}} y_{k}^{\mathrm{I}}+\mu_{k} y_{0}^{\mathrm{I}}\right), \text { for } k=2, \ldots, N,
\end{aligned}\right.
$$

where $x_{0}^{\mathrm{I}}, \ldots, x_{N}^{\mathrm{I}}, y_{0}^{\mathrm{I}}, \ldots, y_{N}^{\mathrm{I}}$ are all independent Gaussian random variables with zero mean and variance of $\frac{1}{2}$, and $\left\{\mu_{k}\right\}$ are the cross-correlation parameters satisfying (7). Note that our model focuses on a typical FAMA user in the presence of interference and omits the user index because the model applies to all users equally if their statistics are identical. ${ }^{4}$

The signal-to-interference plus noise ratio (SINR) at the $k$ th port can be expressed as

$$
\begin{aligned}
\operatorname{SINR}_{k} & \stackrel{(a)}{=} \frac{\left|g_{k}\right|^{2} \mathrm{E}\left[|s|^{2}\right]}{\sum_{i=1}^{N_{\mathrm{I}}}\left|g_{k}^{(i)}\right|^{2} \mathrm{E}\left[\left|s_{i}\right|^{2}\right]+\sigma_{\eta}^{2}} \\
& \stackrel{(b)}{=} \frac{\left|g_{k}\right|^{2} \mathrm{E}\left[|s|^{2}\right]}{\left|g_{k}^{\mathrm{I}}\right|^{2}+\sigma_{\eta}^{2}} \\
& \stackrel{(c)}{=} \frac{\left|g_{k}\right|^{2} \Theta}{\frac{1}{\sigma_{\eta}^{2}}\left|g_{k}^{\mathrm{I}}\right|^{2}+1} \\
& \stackrel{(d)}{\approx} \frac{\left|g_{k}\right|^{2} \mathrm{E}\left[|s|^{2}\right]}{\left|g_{k}^{\mathrm{I}}\right|^{2}} \equiv \operatorname{SIR}_{k},
\end{aligned}
$$

where $(a)$ is a direct result of (9), (b) abuses the notation $g_{k}^{\mathrm{I}}$ to denote a complex Gaussian random variable with zero mean and variance of $\sigma_{\mathrm{I}}^{2}$ with $\left|g_{k}^{\mathrm{I}}\right|^{2}$ representing the instantaneous interference power (averaged over the information symbols of the interferers), (c) uses the definition $\Theta$ and $(d)$ assumes that the interference power is much greater than the noise power. Although SINR is a more accurate performance measure, SIR is a good approximation for interference-limited environments where $N_{\mathrm{I}}$ and/or $\sigma_{\mathrm{I}}$ are large. For optimal performance, it is assumed that the fluid antenna will always switch to the port with the maximum of $\frac{\left|g_{k}\right|}{\left|g_{k}^{1}\right|}$. As a consequence, we are interested in the random variable

$$
g_{\mathrm{FAMA}}=\max \left\{\frac{\left|g_{1}\right|}{\left|g_{1}^{\mathrm{I}}\right|}, \frac{\left|g_{2}\right|}{\left|g_{2}^{\mathrm{I}}\right|}, \ldots, \frac{\left|g_{N}\right|}{\left|g_{N}^{\mathrm{I}}\right|}\right\} .
$$

\footnotetext{
${ }^{4} \mathrm{We}$ do not require the assumption of homogeneous user statistics to conduct our subsequent analysis if $N_{\mathrm{I}}$ is large. In this case, the interferers can have any arbitrary fading distributions and different transmit powers, and the sum of interference will still have a Rayleigh fading envelope due to central limit theorem. The homogeneous user statistics assumption is, however, adopted to simplify our notations and ease our discussion.
}

The channels for the desired signal $\left\{\left|g_{k}\right|\right\}$ and interference $\left\{\left|g_{k}^{\mathrm{I}}\right|\right\}$ are assumed independent but correlated across space, as specified by (7), which causes their ratios to be correlated in a certain way. The random variable $g_{\mathrm{FAMA}}$ corresponds to the square root of the normalized SIR of the received signal.

Instead of considering the outage event based on an SNR target in the single-user case, FAMA focuses on the interference immunity at the users and therefore defines the outage event based on an SIR target, $\bar{\gamma}$ so that

$$
\mathcal{O}_{\text {SIR }}=\left\{g_{\text {FAMA }}^{2}=\max _{k}\left\{\frac{\left|g_{k}\right|^{2}}{\left|g_{k}^{\mathrm{I}}\right|^{2}}\right\}<\frac{\bar{\gamma}}{\mathrm{E}\left[|s|^{2}\right]} \equiv \gamma\right\},
$$

where $\gamma$ is the normalized SIR target.

In the following, we are dedicated to present performance analysis for the FAMA network which will help understand the achievable outage performance and characterize the capacity scaling of FAMA against various system parameters.

\section{MAin RESUlts}

In this section, we present our analysis on the achievable performance of FAMA. We will begin by obtaining the outage probability for the SIR of a FAMA user, and a closed-form outage probability upper bound. Afterwards, we will introduce an average outage capacity lower bound to estimate the overall outage capacity performance of a FAMA network. A definition of multiplexing gain will then be given, from which we will investigate how the system parameters such as the number of ports and size of the fluid antenna impact on the multiplexing gain and capacity performance of the network. To help readers follow our mathematical derivations, some important variables and functions are summarized in TABLE I.

Theorem 1: The outage probability of the SIR, with a target $\gamma$, for a FAMA user is given by (17) (see top of next page), ${ }^{5}$ where $I_{0}(\cdot)$ denotes the zero-order modified Bessel function of the first kind, $Q_{1}(\cdot, \cdot)$ is the first-order Marcum- $Q$ function, and the parameters $\sigma, \sigma_{\mathrm{I}}$ and $\left\{\mu_{k}\right\}$ are defined previously.

Proof: See Appendix A.

Theorem 1 gives out the exact outage probability expression for the SIR at a FAMA user. However, if $z \rightarrow \infty, I_{0}(z) \rightarrow \infty$,

$$
\begin{aligned}
& { }^{5} \text { If SINR is considered, then the outage probability, Prob }(\operatorname{SINR}<\bar{\gamma})= \\
& \operatorname{Prob}\left(\max _{k}\left\{\frac{\left|g_{k}\right|^{2} \Theta}{\frac{1}{\sigma_{\eta}^{2}}\left|g_{k}^{1}\right|^{2}+1}\right\}<\bar{\gamma}\right) \text {, can be derived as } \\
& \operatorname{Prob}(\operatorname{SINR}<\bar{\gamma})=\int_{t_{1}=0}^{\infty} \frac{2 t_{1}}{\sigma_{\mathrm{I}}^{2}} e^{-\frac{t_{1}^{2}}{\sigma_{\mathrm{I}}^{2}}} \int_{t=0}^{\sqrt{\frac{\gamma}{\Theta}\left(\frac{t_{1}^{2}}{\sigma_{\eta}^{2}}+1\right)} e^{-t} \prod_{k=2}^{N}\{} \\
& \int_{t_{k}=0}^{\infty}\left[1-Q_{1}\left(\sqrt{\frac{2 \mu_{k}^{2}}{1-\mu_{k}^{2}}} \sqrt{t}, \sqrt{\frac{2}{\sigma^{2}\left(1-\mu_{k}^{2}\right)}} \sqrt{\frac{\gamma}{\Theta}\left(\frac{t_{k}^{2}}{\sigma_{\eta}^{2}}+1\right)}\right)\right] \\
& \left.\times \frac{2 t_{k}}{\sigma_{\mathrm{I}}^{2}\left(1-\mu_{k}^{2}\right)} e^{-\frac{t_{k}^{2}+\mu_{k}^{2} t_{1}^{2}}{\sigma_{\mathrm{I}}^{2}\left(1-\mu_{k}^{2}\right)}} I_{0}\left(\frac{2 \mu_{k} t_{1} t_{k}}{\sigma_{\mathrm{I}}^{2}\left(1-\mu_{k}^{2}\right)}\right) d t_{k}\right\} d t d t_{1} .
\end{aligned}
$$

To proceed further, we will need to evaluate integral of the form

$$
\int_{0}^{\infty} x e^{-\frac{x^{2}}{2}} I_{0}(c x) Q_{1}\left(b, a_{1} \sqrt{a_{2} x^{2}+1}\right) d x,
$$

which is not known to have a closed-form expression due to the complicated second argument of the Marcum-Q function. 
TABLE I

NOTATIONS OF IMPORTANT VARIABLES AND FUNCTIONS

\begin{tabular}{|c|c|}
\hline Notation & Definition \\
\hline$W \lambda$ & size of fluid antenna where $W$ is the normalized size and $\lambda$ is the wavelength \\
\hline$N$ & number of ports \\
\hline$s$ & information symbol of the user of interest \\
\hline$g_{k}$ & complex channel of the desired signal at the $k$-th port with $\mathrm{E}\left[\left|g_{k}\right|^{2}\right]=\sigma^{2}$ \\
\hline$\eta_{k}$ & complex AWGN at the $k$-th port with zero mean and $\mathrm{E}\left[\left|\eta_{k}\right|^{2}\right]=\sigma_{\eta}^{2}$ \\
\hline$\Theta=\frac{\mathrm{E}\left[|s|^{2}\right]}{\sigma_{\eta}^{2}}$ & symbol power to the noise power ratio \\
\hline$\Gamma=\sigma^{2} \Theta$ & average received SNR \\
\hline$\mu_{k}$ & cross-correlation between the $k$-th port and the first port \\
\hline$J_{0}(z)$ & zero-order Bessel function of the first kind \\
\hline $\bar{\gamma}$ & target SNR for the single-user case (or SIR for FAMA) \\
\hline$\gamma$ & normalized target SNR for the single-user case with $\gamma=\frac{\gamma}{\Theta}$ (or SIR for FAMA with $\gamma=\frac{\gamma}{\mathrm{E}\left[|s|^{2}\right]}$ ) \\
\hline$g_{k}^{(i)}$ & complex channel from the $i$-th interferer at the $k$-th port \\
\hline$s_{i}$ & information symbol of the $i$-th interferer \\
\hline$N_{\mathrm{I}}$ & number of interferers \\
\hline$g_{k}^{\mathrm{I}}$ & complex aggregate interference signal with $\mathrm{E}\left[\left|g_{k}^{1}\right|^{2}\right]=\sigma_{\mathrm{I}}^{2}$ \\
\hline$I_{0}(z)$ & zero-order modified Bessel function of the first kind \\
\hline$Q_{1}(a, b)$ & the first-order Marcum-Q function \\
\hline $\operatorname{Prob}\left(\mathcal{O}_{\mathrm{SNR}}\right)$ & SNR-based outage probability for the single-user system with $\mathcal{O}_{\mathrm{SNR}}=\{\mathrm{SNR}<\bar{\gamma}\}=\left\{\max _{k}\left\{\left|g_{k}\right|^{2}\right\}<\gamma\right\}$ \\
\hline $\operatorname{Prob}\left(\mathcal{O}_{\text {SIR }}\right)$ & SIR-based outage probability for FAMA with $\mathcal{O}_{\mathrm{SIR}}=\{\mathrm{SIR}<\bar{\gamma}\}=\left\{g_{\mathrm{FAMA}}^{2}=\max _{k}\left\{\frac{\left|g_{k}\right|^{2}}{\left|g_{k}^{1}\right|^{2}}\right\}<\gamma\right\}$ \\
\hline$\varepsilon_{\mathrm{UB}}(\gamma)$ & generic upper bound for the outage probability \\
\hline$\varepsilon_{\mathrm{UB}}^{\mathrm{I}}(\gamma)$ & the upper bound for the outage probability in (19) \\
\hline$\varepsilon_{\mathrm{UB}}^{11}(\gamma)$ & the upper bound for the outage probability in (20) \\
\hline$E_{k}(z)$ & generalized exponential integral \\
\hline$C_{\text {FAMA }}(\gamma)$ & average network outage rate for FAMA in (21) \\
\hline$C_{\mathrm{LB}}(\gamma)$ & lower bound for the average network outage rate for FAMA in (22) \\
\hline$m$ & multiplexing gain in (23) \\
\hline$\underline{m}$ & lower bound for the multiplexing gain in (24) \\
\hline$J_{0}^{-1}(\mu)$ & inverse of $J_{0}(\cdot)$ such that $\rho^{*}=J_{0}^{-1}\left(\mu^{*}\right)$ returns the minimum value of $\rho^{*}$ to ensure that $\left|J_{0}(\rho)\right| \leq \mu^{*}$ for $\rho \geq \rho^{*}$ \\
\hline
\end{tabular}

$$
\begin{aligned}
\operatorname{Prob}(\operatorname{SIR}<\bar{\gamma}) & =\int_{0}^{\infty} e^{-z} \int_{0}^{\frac{\sigma_{\mathrm{I}}^{2} \gamma}{\sigma^{2}} z} e^{-t}\left\{\prod _ { k = 2 } ^ { N } \left[1+\left(\frac{\frac{\sigma_{\mathrm{I}}^{2} \gamma}{\sigma^{2}}}{\frac{\sigma_{\mathrm{I}}^{2} \gamma}{\sigma^{2}}+1}\right) e^{-\left(\frac{1}{\frac{\sigma_{1}^{2} \gamma}{\sigma^{2}}+1}\right) \frac{\mu_{k}^{2}}{1-\mu_{k}^{2}}\left(\frac{\sigma_{\mathrm{I}}^{2} \gamma}{\sigma^{2}} z+t\right)} \times\right.\right. \\
& \left.\left.I_{0}\left(\frac{\frac{\sigma_{\mathrm{I}} \sqrt{\gamma}}{\sigma}}{\frac{\sigma_{\mathrm{I}}^{2} \gamma}{\sigma^{2}}+1}\left(\frac{2 \mu_{k}^{2}}{1-\mu_{k}^{2}}\right) \sqrt{z t}\right)-Q_{1}\left(\frac{1}{\sqrt{\frac{\sigma_{\mathrm{I}}^{2} \gamma}{\sigma^{2}}+1}} \sqrt{\frac{2 \mu_{k}^{2}}{1-\mu_{k}^{2}}} \sqrt{t}, \sqrt{\frac{\frac{\sigma_{\mathrm{I}}^{2} \gamma}{\sigma^{2}}}{\frac{\sigma_{\mathrm{I}}^{2} \gamma}{\sigma^{2}}+1}} \sqrt{\frac{2 \mu_{k}^{2}}{1-\mu_{k}^{2}}} \sqrt{z}\right)\right]\right\} d t d z,
\end{aligned}
$$

which happens when evaluating (17). To get around this, the second term inside the product can be evaluated by (18) (see next page), which can be obtained from the definition of $I_{0}(\cdot)$.

Recognize that the expression (17) includes all the essential system information such as the average aggregate interference power $\sigma_{\mathrm{I}}^{2}$, the channel power $\sigma^{2}$, the number of ports $N$ of the fluid antenna, and the cross-correlation of the ports $\left\{\mu_{k}\right\}$ while the signal power $\mathrm{E}\left[|s|^{2}\right]$ is absorbed in the normalized SIR target $\gamma$. However, although numerical integration for (17) is possible, the computational complexity is very high when $N$ is large, and the integral form also does not permit any insight to be gained. As a result, we obtain two outage probability upper bounds, which we present in the next theorems.

Theorem 2: The SIR outage probability expression in (17) is upper bounded by (19) (see next page).

Proof: See Appendix B.

Corollary 1: As $N \rightarrow \infty$, the SIR outage probability goes to 0 as long as $\left|\mu_{k}\right| \neq 1 \forall k$.
Proof: It can be observed from (19) that the integrand is a product of $N+1$ less-than-one numbers. If $N \rightarrow \infty$, it therefore goes to 0 which completes the proof.

Theorem 2 appears to identify the benefit of each individual port in reducing the outage probability and Corollary 1 confirms the feasibility of FAMA since the interference at the MS can always be avoided if $N$ is large enough. Also, (19) further allows a closed-form outage probability upper bound to be derived for the special case where $\left|\mu_{2}\right|=\cdots=\left|\mu_{N}\right|=\mu$. This result is presented in the following theorem.

Theorem 3: If $\left|\mu_{2}\right|=\cdots=\left|\mu_{N}\right|=\mu$, then the SIR outage probability is upper bounded by

$$
\begin{aligned}
\operatorname{Prob}(\operatorname{SIR} & <\bar{\gamma}) \lesssim \varepsilon_{\mathrm{UB}}^{\mathrm{II}}(\gamma)=\frac{1-\mu^{2}}{4 \mu^{2}} e^{\frac{1-\mu^{2}}{4 \mu^{2}}} \times \\
& \sum_{k=0}^{N-1} \frac{\left(\begin{array}{c}
N-1 \\
k
\end{array}\right)(-1)^{k}}{\left(\frac{\sigma_{\mathrm{I}}^{2} \gamma}{\sigma^{2}}\right)^{k}} e^{\frac{k}{4}} E_{k}\left(\frac{k}{4}+\frac{1-\mu^{2}}{4 \mu^{2}}\right),
\end{aligned}
$$




$$
\begin{aligned}
\left(\frac{\frac{\sigma_{\mathrm{I}}^{2} \gamma}{\sigma^{2}}}{\frac{\sigma_{\mathrm{I}}^{2} \gamma}{\sigma^{2}}+1}\right) e^{-\left(\frac{1}{\frac{\sigma_{\mathrm{I}}^{2} \gamma}{\sigma^{2}}+1}\right) \frac{\mu_{k}^{2}}{1-\mu_{k}^{2}}\left(\frac{\sigma_{\mathrm{I}}^{2} \gamma z}{\sigma^{2}}+t\right)} I_{0}\left(\frac{\frac{\sigma_{\mathrm{I}} \sqrt{\gamma}}{\sigma}}{\frac{\sigma_{\mathrm{I}}^{2} \gamma}{\sigma^{2}}+1}\left(\frac{2 \mu_{k}^{2}}{1-\mu_{k}^{2}}\right) \sqrt{z t}\right) \\
=\frac{1}{\pi}\left(\frac{\frac{\sigma_{\mathrm{I}}^{2} \gamma}{\sigma^{2}}}{\frac{\sigma_{\mathrm{I}}^{2} \gamma}{\sigma^{2}}+1}\right) e^{-\left(\frac{1}{\frac{\sigma_{\mathrm{I}}^{2} \gamma}{\sigma^{2}}+1}\right) \frac{\mu_{k}^{2}}{1-\mu_{k}^{2}}\left(\frac{\sigma_{\mathrm{I}} \sqrt{\gamma z}}{\sigma}-\sqrt{t}\right)^{2}} \int_{0}^{\pi} e^{-\left(\frac{1}{\frac{\sigma_{\mathrm{I}}^{2} \gamma}{\sigma^{2}}+1}\right) \frac{\mu_{k}^{2}}{1-\mu_{k}^{2}} \frac{4 \sigma_{\mathrm{I}} \sqrt{\gamma z t}}{\sigma} \sin ^{2} \frac{\theta}{2}} d \theta
\end{aligned}
$$

$$
\operatorname{Prob}(\mathrm{SIR}<\bar{\gamma}) \leq \varepsilon_{\mathrm{UB}}^{\mathrm{I}}(\gamma)=\int_{0}^{\infty} e^{-z}\left(1-e^{-\frac{\sigma_{\mathrm{I}}^{2} \gamma}{\sigma^{2}} z}\right) \prod_{k=2}^{N}\left[1-\left(\frac{1}{\frac{\sigma_{\mathrm{I}}^{2} \gamma}{\sigma^{2}}+1}\right) \frac{e^{-\left(\frac{1}{\frac{\sigma_{\mathrm{I}}^{2} \gamma}{\sigma^{2}}+1}\right)\left(\frac{\mu_{k}^{2}}{1-\mu_{k}^{2}}\right) \frac{\sigma_{\mathrm{I}}^{2} \gamma}{\sigma^{2}} z}}{\left.1+4\left(\frac{1}{\frac{\sigma_{\mathrm{I}}^{2} \gamma}{\sigma^{2}}+1}\right)\left(\frac{\mu_{k}^{2}}{1-\mu_{k}^{2}}\right) \frac{\sigma_{\mathrm{I}}^{2} \gamma}{\sigma^{2}} z\right] d z}\right.
$$

where $E_{k}(\cdot)$ denotes the generalized exponential integral.

Proof: See Appendix C.

The following theorem quantifies the average network outage rate performance of the FAMA network, which quantifies how much the improved outage performance is translated into the gain in capacity on the average sense, if fixed-rate encoders are used at the BSs. The result also provides a lower-bound estimate of the capacity using the bounds derived above.

Theorem 4: The average network outage rate of FAMA is given by

$$
\begin{aligned}
& C_{\mathrm{FAMA}}(\gamma) \\
& \approx\left(N_{\mathrm{I}}+1\right)(1-\operatorname{Prob}(\mathrm{SIR}<\bar{\gamma})) \log _{2}(1+\bar{\gamma}) \\
& \geq\left(N_{\mathrm{I}}+1\right)\left(1-\varepsilon_{\mathrm{UB}}(\gamma)\right) \log _{2}(1+\bar{\gamma}) \equiv C_{\mathrm{LB}}(\gamma),
\end{aligned}
$$

where $\varepsilon_{\mathrm{UB}}(\gamma)$ can be either $\varepsilon_{\mathrm{UB}}^{\mathrm{I}}(\gamma)$ in (19) or $\varepsilon_{\mathrm{UB}}^{\mathrm{II}}(\gamma)$ in (20).

Proof: The capacity expression in (21) follows directly from the definition of average outage rate in [39] with our approximation $\mathrm{SINR} \approx \mathrm{SIR}$. This corresponds to the system in which each BS transmitter transmits a constant coding rate $\log _{2}(1+\bar{\gamma})$ which is successfully decoded with probability $1-\operatorname{Prob}(\operatorname{SIR}<\bar{\gamma})$. For the FAMA network, as all the users are independent, the average outage rate of the whole network is therefore the average outage rate for a typical user scaled by the total number of users. Replacing the probability by an outage probability upper bound finally gives (22).

For multiple access, it is particularly important to characterize the capacity scaling using the definition of multiplexing gain. This is formally established below.

Definition 1: The multiplexing gain for the FAMA network is defined as

$$
\begin{aligned}
m & \triangleq\left(N_{\mathrm{I}}+1\right)(1-\operatorname{Prob}(\operatorname{SIR}<\bar{\gamma})) \\
& \geq\left(N_{\mathrm{I}}+1\right)\left(1-\varepsilon_{\mathrm{UB}}(\gamma)\right) \equiv \underline{m} .
\end{aligned}
$$

The above definition follows naturally when finding the ratio between the network outage rate of FAMA and the rate of a single user occupying the same amount of bandwidth, i.e., $\log _{2}(1+\bar{\gamma})$. As a result, $m$ finds the capacity scaling of the network using fluid antennas for multiple access. Normally, if we overlap users without any interference avoidance mechanism,
$\operatorname{Prob}(\operatorname{SIR}<\bar{\gamma}) \approx 1$ for any meaningful $\bar{\gamma}$, and $m \approx 0$, which would be worse than single-user communications. In FAMA, however, the fluid antennas decrease the outage probability by switching to the ports where their interference undergoes a deep fade, achieving their maximum SIRs. Admittedly, the definition has assumed that the users are statistically identical and have the same SIR target. In Appendix D, we discuss the extension to the case of non-homogeneous users.

The following theorem attempts to unpack the multiplexing gain of FAMA against the system parameters using the bound $\varepsilon_{\mathrm{UB}}^{\mathrm{II}}(\gamma)$ in (20) and under the uniform correlation assumption.

Theorem 5: FAMA achieves a multiplexing gain of $m$ or more $\left(\leq N_{\mathrm{I}}+1\right)$ with a normalized target SIR $\gamma$ if $N$ satisfies

$$
\begin{aligned}
& \frac{1-\mu^{2}}{4 \mu^{2}} e^{\frac{1-\mu^{2}}{4 \mu^{2}}} \sum_{k=0}^{N-1} \frac{\left(\begin{array}{c}
N-1 \\
k
\end{array}\right)(-1)^{k}}{\left(\frac{\sigma_{\mathrm{I}}^{2} \gamma}{\sigma^{2}}\right)^{k}} e^{\frac{k}{4}} E_{k}\left(\frac{k}{4}+\frac{1-\mu^{2}}{4 \mu^{2}}\right) \\
& \leq 1-\frac{m}{N_{\mathrm{I}}+1},
\end{aligned}
$$

which, under the condition that $\mu$ is reasonably small, further yields the simplified condition

$$
\sum_{k=1}^{N-1} \frac{\left(\begin{array}{c}
N-1 \\
k
\end{array}\right)(-1)^{k+1}}{\left(\frac{\sigma_{\mathrm{I}}^{2} \gamma}{\sigma^{2}}\right)^{k}}\left(1-k \mu^{2}\right) \gtrsim \frac{m}{N_{\mathrm{I}}+1} .
$$

For an ambitious target $\gamma$ and very large interference power $\sigma_{\mathrm{I}}^{2}$, i.e., $\frac{\sigma_{\mathrm{I}}^{2} \gamma}{\sigma^{2}}$ is very large, we have

$$
N \gtrsim\left(\frac{m}{N_{\mathrm{I}}+1}\right) \frac{\left(\frac{\sigma_{\mathrm{I}}^{2} \gamma}{\sigma^{2}}\right)}{1-\mu^{2}}+1 \approx \frac{m \gamma}{1-\mu^{2}}+1,
$$

where the last result arises when $\sigma_{\mathrm{I}}^{2}=N_{\mathrm{I}} \sigma^{2}$ and $N_{\mathrm{I}}$ is large.

Proof: See Appendix E.

Notice that the power of the aggregate interference $\sigma_{\mathrm{I}}^{2}$ could be linked to the number of interferers occupying in the same environment. If all the interferers have the same power and that each of their power is identical to that of the desired user, then $\sigma_{\mathrm{I}}^{2}=N_{\mathrm{I}} \sigma^{2}$, and $\frac{\sigma_{\mathrm{I}}^{2} \gamma}{\sigma^{2}}=N_{\mathrm{I}} \gamma$. The condition (27) makes sense as $N$ appears to be directly proportional to the multiplexing gain and the target SIR. In addition, high cross-correction $\mu$ 
will require a larger $N$ to compensate for the performance. The next corollary explicitly illustrates how the achievable multiplexing gain scales with the system parameters.

Corollary 2: The multiplexing gain of the FAMA network is bounded by

$$
\begin{aligned}
N_{\mathrm{I}}+1 \geq m & \gtrsim \min \left\{\frac{(N-1)\left(1-\mu^{2}\right)\left(N_{\mathrm{I}}+1\right)}{\left(\frac{\sigma_{\mathrm{I}}^{2} \gamma}{\sigma^{2}}\right)}, N_{\mathrm{I}}+1\right\} \\
& \approx \min \left\{\frac{(N-1)\left(1-\mu^{2}\right)}{\gamma}, N_{\mathrm{I}}+1\right\} .
\end{aligned}
$$

Proof: The upper bound of $m$ comes directly from (24). In order to obtain the lower bound, we use the outage probability upper bound $\varepsilon_{\mathrm{UB}}^{\mathrm{II}}(\gamma)$ in (20) which as shown in Appendix E can be approximated as $\varepsilon_{\mathrm{UB}}^{\mathrm{II}}(\gamma) \approx 1-(N-1)\left(1-\mu^{2}\right) \frac{\sigma^{2}}{\sigma_{\mathrm{I}}^{2} \gamma}$. The lower bound in (28) is then obtained by ensuring that the lower bound does not exceed $N_{\mathrm{I}}+1$.

In terms of the SIR outage probability, a FAMA network serving two users each achieving a target SIR $N_{\mathrm{I}} \gamma$ is equivalent to the FAMA network with $N_{\mathrm{I}}+1$ users, each achieving a target SIR $\gamma$. The following theorem illustrates the intriguing fact that FAMA prefers serving more users with a less SIR target than serving less users with a larger SIR target as far as the average network outage capacity is concerned.

Theorem 6: FAMA obtains higher capacity by serving more users, with a less SIR target because

$$
\frac{\left.C_{\text {FAMA }}(\gamma)\right|_{N_{\mathrm{I}}+1}}{\left.C_{\text {FAMA }}\left(N_{\mathrm{I}} \gamma\right)\right|_{2}} \approx\left(\frac{N_{\mathrm{I}}+1}{2}\right) \frac{1}{1+\log _{\gamma} N_{\mathrm{I}}},
$$

in which $\left.C_{\mathrm{FAMA}}(\gamma)\right|_{M}$ denotes the average network outage rate with $M$ simultaneous FAMA users each achieving a large normalized target SIR of $\gamma$.

Proof: At the user level, the outage probability is the same for the two cases: one that has two coexisting users each with a target SIR $N_{\mathrm{I}} \gamma$ and another that has $N_{\mathrm{I}}+1$ coexisting users but each with a target SIR $\gamma$. Consequently, the capacity ratio gives (29), which completes the proof.

For example, if $\gamma=10$ and $N_{\mathrm{I}}=100, \frac{\left.C_{\mathrm{FAMA}}(\gamma)\right|_{N_{\mathrm{I}}+1}}{\left.C_{\mathrm{FAMA}}\left(N_{\mathrm{I}} \gamma\right)\right|_{2}} \approx 17$, meaning that we can have 17 times more capacity for serving 101 users each with a target SIR of 10 than two users with a target SIR of 1000. The gain is not surprising because SIR is inside the $\log$ of the capacity formula while the number of users scales the capacity directly. What is less intuitive is how the excessive inter-user interference gets resolved when $N_{\text {I }}$ is large to sustain the overall capacity rise. Under the notion of utilizing deep fades for multiple access, it is actually desirable to have superposition of a larger number of user signals, as this can be translated into deeper fades on the signal envelope and more opportunity for multiple access. However, if $N_{\mathrm{I}}$ increases, it usually comes with an increase in the total interference power which is indeed undesirable.

The following results aim to understand how the size of the fluid antenna affects the achievable performance. The first result below is obtained based on the uniform cross-correlation assumption with $\mu$. More general results then follow.

Corollary 3: For a sufficiently large but fixed $N$ at every FAMA user, FAMA achieves the multiplexing gain $m$ or more $\left(\leq N_{\mathrm{I}}+1\right)$ with a normalized target SIR $\gamma$ if the crosscorrelation parameter at the FAMA user satisfies

$$
\begin{aligned}
\mu \leq \mu^{*}= & \sqrt{\frac{\sum_{k=1}^{N-1} \frac{\left(\begin{array}{c}
N-1 \\
k
\end{array}\right)(-1)^{k+1}}{\left(\frac{\sigma_{\mathrm{I}}^{2} \gamma}{\sigma^{2}}\right)^{k}}-\frac{m}{N_{\mathrm{I}}+1}}{\sum_{k=1}^{N-1} \frac{k\left(\begin{array}{l}
N-1 \\
k
\end{array}\right)(-1)^{k+1}}{\left(\frac{\sigma_{\mathrm{I}}^{2} \gamma}{\sigma^{2}}\right)^{k}}}} \\
& \approx \sqrt{1-\frac{m\left(\frac{\sigma_{\mathrm{I}}^{2} \gamma}{\sigma^{2}}\right)}{\left(N_{\mathrm{I}}+1\right)(N-1)}} .
\end{aligned}
$$

Proof: This comes directly from (26) and (27).

A few intuitive observations can be made from (30). First of all, more demanding targets $\gamma$ and $m$ would require $\mu$ to be smaller, as expected. Also, the increasing number of ports $N$ has the impact of lessening the requirement on the spatial correlation. On the other hand, very interestingly, if the number of interferers $N_{\mathrm{I}}$ increases while keeping the total power $\sigma_{\mathrm{I}}^{2}$ fixed, it has a desirable effect on relaxing the requirement on $\mu$. This can be explained by the fact that $N_{\mathrm{I}}$ contributes to more multipath which in turn gives rise to more fluctuations on the signal envelope that can be exploited by FAMA.

Thus far, some physical insight has been gained but under the assumption that all the cross-correlation parameters are the same, i.e., $\left|\mu_{2}\right|=\cdots=\left|\mu_{N}\right|=\mu$, which unfortunately may not correspond to any real configuration. The next theorems address the general case, and link the dimension of the fluid antenna, $W \lambda$, at a FAMA user to other system parameters.

Theorem 7: The minimum required size of the fluid antenna at each FAMA user to achieve the targets $m\left(\leq N_{\mathrm{I}}+1\right)$ and $\gamma$ for a given $N_{\text {I }}$ and large but fixed $N$ is given by

$$
W \geq \frac{1}{\pi} J_{0}^{-1}\left(\sqrt{1-\frac{m\left(\frac{\sigma_{\mathrm{I}}^{2} \gamma}{\sigma^{2}}\right)}{\left(N_{\mathrm{I}}+1\right)\left(\left\lfloor\frac{N}{2}\right\rfloor-1\right)}}\right),
$$

where $J_{0}^{-1}(\cdot)$ computes the inverse of $J_{0}(\cdot)$ and is defined in such a way that $\rho^{*}=J_{0}^{-1}\left(\mu^{*}\right)$ returns the minimum value of $\rho^{*}$ to ensure that $\left|J_{0}(\rho)\right| \leq \mu^{*}$ for $\rho \geq \rho^{*}$. Additionally, $\lfloor\cdot\rfloor$ returns the largest integer that is smaller than the input.

Proof: See Appendix F.

Theorem 8: For the general case, the number of ports $N$ for each FAMA user should satisfy

$$
N \geq 2\left[\frac{m\left(\frac{\sigma_{\mathrm{I}}^{2} \gamma}{\sigma^{2}}\right)}{\left(N_{\mathrm{I}}+1\right)\left(1-J_{0}^{2}(\pi W)\right)}+1\right],
$$

for $m \leq N_{\mathrm{I}}+1$.

Proof: This result is obtained by changing the subject of the condition (31) as $N$.

From (32), we can observe that the power of FAMA comes predominately from the number of ports, $N$, though $W$ has an impact on the required value of $N$ to meet the SIR target $\gamma$ and the multiplexing gain $m$. The result in Theorem 8 suggests that theoretically, as long as $W \neq 0$, there always exists a finite value of $N$ such that FAMA achieves any arbitrarily given multiplexing gain $m$ with any given target SIR $\gamma$. On a negative note, however, the required value of $N$ tends to be 
TABLE II

SimUlation PARAMETERS

\begin{tabular}{c|l}
\hline Parameter & Value \\
\hline \hline Normalized size of fluid antenna, $W$ & $0.5,2$ \\
\hline Number of ports, $N$ & $10,20,50, \ldots, 250, \ldots, 3000$ \\
\hline Average symbol power, $\mathrm{E}\left[|s|^{2}\right]$ & 1 \\
\hline Target SIR, $\bar{\gamma}=\gamma$ & $0-20 \mathrm{~dB}$ \\
\hline Number of interferers, $N_{\mathrm{I}}$ & $1,2,3, \ldots, 200$ \\
\hline Number of FAMA users, $N_{\mathrm{I}}+1$ & $2,3,4, \ldots, 201$ \\
\hline Multiplexing gain lower bound, $\underline{m}$ & $1-90$ \\
\hline
\end{tabular}

quite large. For example, assuming $\sigma_{\mathrm{I}}^{2}=N_{\mathrm{I}} \sigma^{2}$, even if $W$ is so large that $J_{0}(\pi W) \approx 0$, according to (32), we still need

$$
N \approx 2 m \gamma+2=2(2)(10)+2=42 \text { if } m=2 \text { and } \gamma=10 .
$$

The above estimate is, however, based on a sufficient condition and the outage probability upper bound. Therefore, the actual minimum required $N$ is expected to be smaller.

Corollary 4: The multiplexing gain of the FAMA network for the general case is lower bounded by

$$
\begin{aligned}
m & \gtrsim \min \left\{\frac{\left(\left\lfloor\frac{N}{2}\right\rfloor-1\right)\left(1-J_{0}^{2}(\pi W)\right)\left(N_{\mathrm{I}}+1\right)}{\left(\frac{\sigma_{\mathrm{I}}^{2} \gamma}{\sigma^{2}}\right)}, N_{\mathrm{I}}+1\right\} \\
& \approx \min \left\{\frac{\left(\left\lfloor\frac{N}{2}\right\rfloor-1\right)\left(1-J_{0}^{2}(\pi W)\right)}{\gamma}, N_{\mathrm{I}}+1\right\} .
\end{aligned}
$$

Proof: The result is obtained from (28) by using the same argument used in Appendix F.

Corollary 4 illustrates conservatively the potential capability of FAMA whose average outage capacity scales linearly with the number of ports, $N$, as long as $m \leq N_{\mathrm{I}}+1$ according to the definition (24), but is discounted by the SIR target $\gamma$. The size of fluid antenna, $W$, dictates the amount of spatial correlation that has a critical impact on $m$ only if $W$ is very small. For example, if $W=0.5, J_{0}^{2}(0.5 \pi) \approx 0.22$ which is already small, and will not be a major factor for the capacity performance. This reveals that space being the major limitation due to spatial correlation may be overstated, and massive capacity increase is possible as long as $N$ can grow to be large.

\section{NumericAl Results}

In this section, we provide numerical results to help demonstrate the potential for FAMA. All the results were obtained using the expressions derived throughout this paper. As there are many system parameters involved, many results are presented but our aim is to highlight some key observations. The results are meant to provide insight on the possible performance of FAMA from an information-theoretic perspective and may not represent an accurate evaluation of FAMA in practice as there are factors relating to the implementation of fluid antenna that should be considered in the future. That said, our results can be interpreted as some conservative bounds that can provide meaningful indication of the achievable performance. In the results, we have assumed that all the MS users are statistically identical and that $\sigma_{\mathrm{I}}^{2}=N_{\mathrm{I}} \sigma^{2}$ for ease of discussion. To aid the readers, TABLE II lists the parameters considered.

\section{A. Results and Discussion}

Results in Fig. 2 compare the exact SIR outage probability in (17) and the upper bound in (19) for different settings. It can be seen that the upper bound is not particularly tight but can be interpreted as a conservative measure to the achievable performance of FAMA and thus used as a means to understand analytically the interplay between different system parameters. In particular, the upper bound will be tighter if the size of fluid antenna, $W$, is larger, or the operating conditions are more challenging, e.g., with a larger number of interferers $N_{\mathrm{I}}$ and/or a larger SIR target $\gamma$. It should be noted that in terms of the network outage rate, the bound always overestimates the required number of ports, $N$, by a margin. Interestingly, the results in Fig. 2(b) show some promising results even if $N$ is not so large. It is observed that with $W=2, N=20$ and $N_{\text {I }}=5$ (i.e., supporting 6 users), the SIR outage probability at a FAMA user is about $30 \%$, which means that FAMA achieves a multiplexing gain of $6 \times(1-0.3)=4.2$. If $N=30$, then the multiplexing gain will be increased to 4.8 .

In Figs. 3-5, the numerical results are provided using the upper bound (19) since the complexity to evaluate (17) can quickly become unaffordable for large $N$. Results in Fig. 3 present the average network outage rate of FAMA when each user has an SIR target of 10dB. As can be observed, a larger $W$ will achieve higher capacity but the difference gradually disappears if $N$ becomes very large. Increasing the number of ports, $N$, clearly enhances capacity but the gain will saturate at some point which depends on the total number of users, $N_{\mathrm{I}}+1$. In particular, the maximum average outage rate is $\left(N_{\mathrm{I}}+1\right) \log _{2}(1+\gamma)$ which is achievable when $N \rightarrow \infty$ (so achieving zero outage probability). As expected, we observe that if $N_{\mathrm{I}}$ increases, then FAMA will deliver greater capacity because the interference at each user can be resolved by fluid antenna with a sufficiently large number of ports, $N$.

We study the impact of the target SIR $\gamma$ through the lower bound $C_{\mathrm{LB}}(\gamma)$ in Fig. 4. The results illustrate that for large $N$, there is an optimal $\gamma$ that maximizes the average network outage rate. A small $\gamma$ may unnecessarily limits the achievable performance for each user but if $\gamma$ is too large, then the outage probability will increase drastically and reduce the network outage rate. Also, the number of interferers $N_{\mathrm{I}}$ generally has a positive impact on the outage rate as discussed above but if $\gamma$ is too large for a given $N$, the interference will harm the overall capacity. Such turning point appears to happen at a larger value of $\gamma$ if $N$ is greater. Despite this, after the turning point, the network outage rate is not much affected by the number of interferers. For $\gamma=0 \mathrm{~dB}$ and $N=20$, a FAMA network with 101 users can deliver a multiplexing gain of 13 (note that $\log _{2}(1+\gamma)=1$ in this case) which will be doubled to 26 as a result of reduction in the SIR outage probability if $N$ is increased to 50 . Therefore, a massive capacity gain is possible using FAMA even for not so large $N$.

The impact of the number of interferers, $N_{\mathrm{I}}$, on the capacity performance is further examined in Fig. 5 where $\gamma=10 \mathrm{~dB}$ is considered. Results indicate that when $N$ is too small, the outage capacity first decreases and then remains the same as $N_{\text {I }}$ increases. For larger values of $N$, in contrast, the outage 


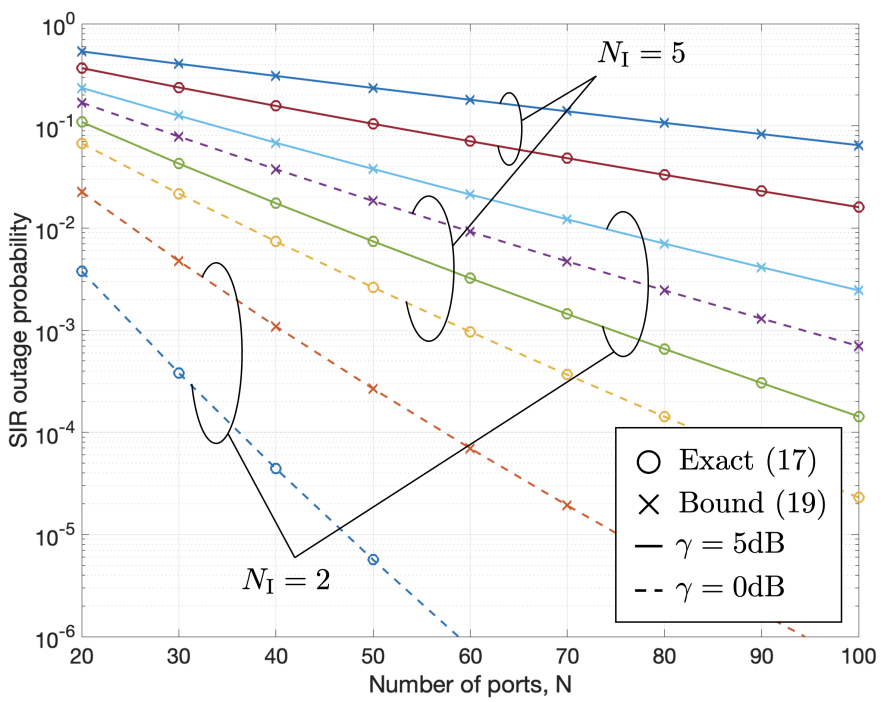

(a) $W=0.5$

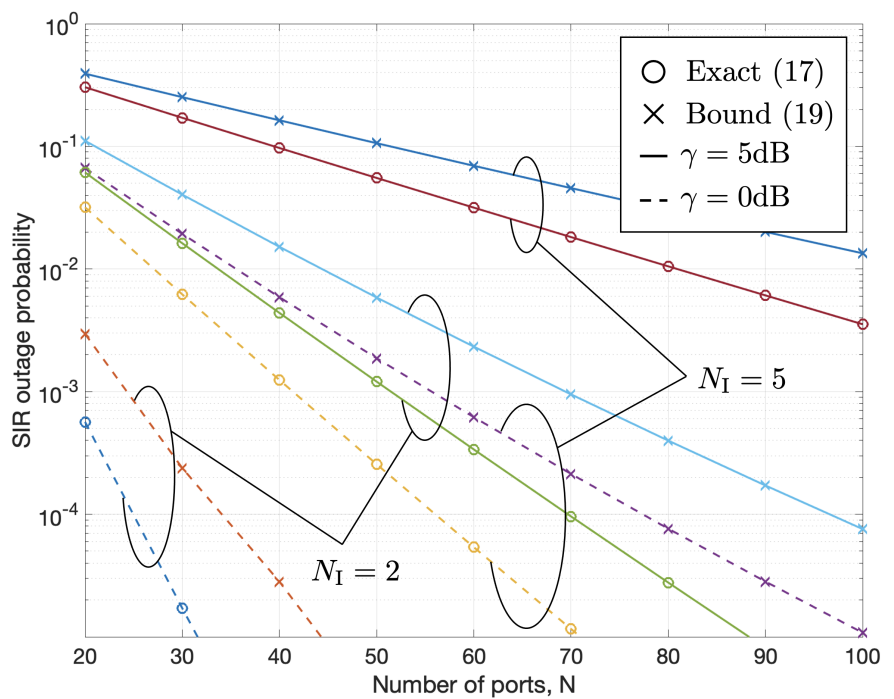

(b) $W=2$

Fig. 2. The exact SIR outage probability (17) versus the upper bound (19).

capacity grows when $N_{\mathrm{I}}$ increases but saturates at some point because the outage capacity gain by the increase in the number of users is then cancelled by the corresponding increase in the outage probability at each user when $N$ is fixed.

Results in Fig. 6 study the multiplexing gain of the FAMA network against the size of fluid antenna at each user via the lower bound $\underline{m}$ using $\varepsilon_{\mathrm{UB}}^{\mathrm{I}}(\gamma)$ in (19). The results illustrate that the impact of $W$ is more obvious when $N$ is larger. As expected, if $W$ increases, the multiplexing gain will follow. The capacity benefit is sharper before $\frac{\lambda}{2}$ but after $\frac{\lambda}{2}$, the increase in the multiplexing gain diminishes. Putting implementation issues aside, this suggests that $W=\frac{\lambda}{2}$ be the smallest size that can obtain the most benefit of fluid antenna. Furthermore,

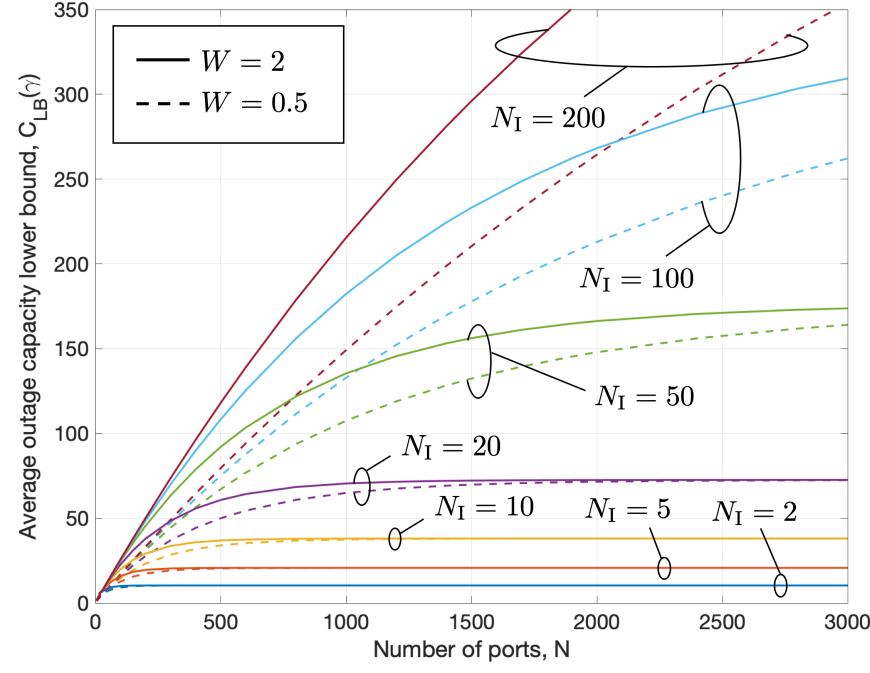

Fig. 3. The capacity lower bound in (22) versus $N$ when $\gamma=10 \mathrm{~dB}$.

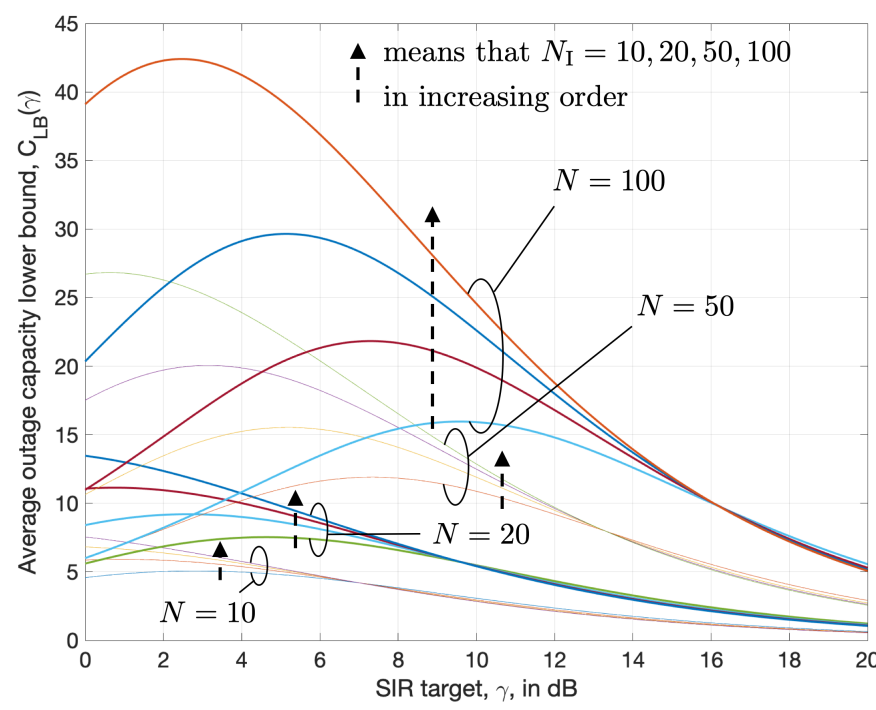

Fig. 4. Capacity lower bound in (22) versus the SIR target $\gamma$ when $W=2$.

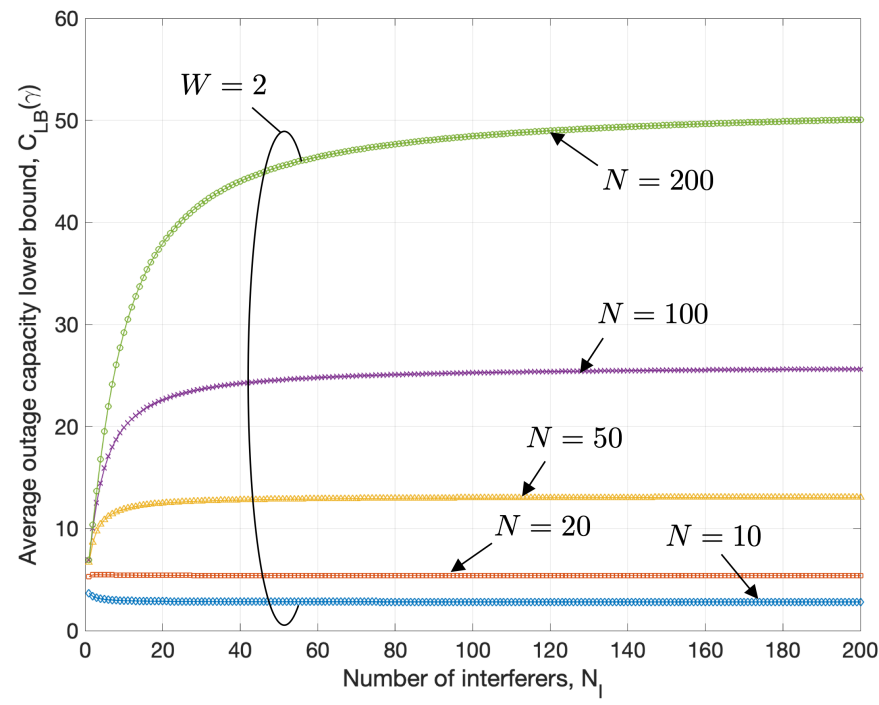

Fig. 5. Capacity lower bound in (22) versus $N_{\mathrm{I}}$ when $\gamma=10 \mathrm{~dB}$. 


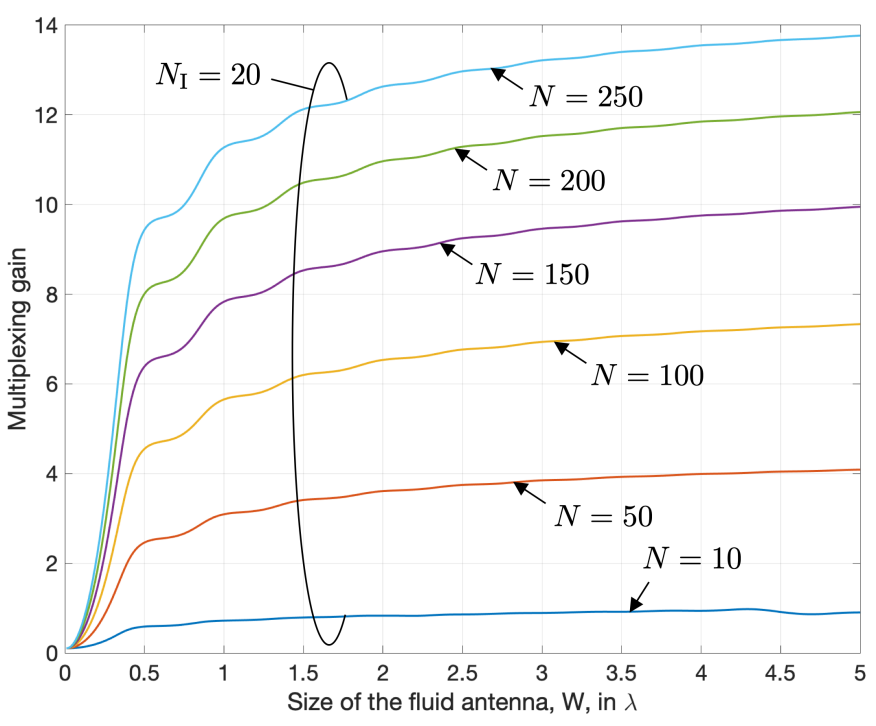

Fig. 6. The multiplexing gain lower bound, $\underline{m}$, in (24) versus the size of the fluid antenna, $W$, when $\gamma=10 \mathrm{~dB}$.

some ripples are observed on the shape of the curves and they come naturally from how the cross-correlation varies over a linear distance which follows from the Bessel function (5). In addition, the results again show that as $N$ increases, a higher multiplexing gain can be achieved. The results also suggest that $N$ be a more important factor than $W$.

Results in Fig. 7 investigate the required number of ports against the multiplexing gain lower bound. First, it should be noted that the multiplexing gain is upper bounded by $N_{\mathrm{I}}+1$ (the number of FAMA users) which is achievable only when the outage probability goes to zero if $N \rightarrow \infty$. The same can be observed when $N$ continues to increase while keeping

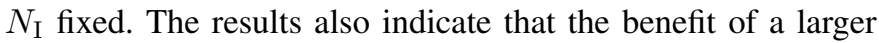
$W$ gets smaller as $N$ can be increased to compensate for the loss of outage probability performance. Another interesting observation is that if $N$ can be extremely large, then FAMA can easily accommodate hundreds of users, all by a single fluid antenna with a reasonable size at each user, and obtain a massive multiplexing gain; see also Fig. 3. Furthermore, we can see the results for more practical values of $N$ from the magnified figure, which reveals that as large as a multiplexing gain of 10 is possible when $N=150$. Note that the results are based on the outage probability upper bound which will overestimate the value of $N$ so it is anticipated that a smaller $N$ will likely achieve the impressive multiplexing gain.

We now take a closer look at the required size of the fluid antenna for each FAMA user as a function of $N$ to achieve a certain multiplexing gain of the network. The results are shown in Fig. 8, which indicates that if $N$ is too small, then it is impossible to have a feasible size $W$ that can achieve a given multiplexing gain. Nonetheless, once $N$ is sufficiently large, $W$ goes down sharply and then gradually decreases as $N$ continues to increase. Results also illustrate that the number of interferers does not have a significant impact on $W$ and $W$ is mainly dependent on the required multiplexing gain. In addition, $N$ needs an exponential increase in values to reduce the required size of the fluid antenna beyond $\frac{\lambda}{2}$.

\section{B. Further Discussion}

The above results are encouraging since multiple access for a large number of users is possible, all by a single-RF chain fluid antenna at each user without coordination amongst the users, CSI at the BS side nor precoding. This conclusion is also drawn from the derived information-theoretic bounds that are supposed to be conservative based on the model. However, it is worth mentioning that there are a couple of assumptions attached to our analysis. First of all, the radiating element of the fluid antenna is modelled as a point. This idealization helps simplify our model greatly but ignores the physical dimension of the element and its RF responses with the device structure. In addition, the radiation pattern of the fluid element is hardly omnidirectional in practice and depends on the shape of the element. This will affect the distribution of the fading envelope and limit the available diversity. As a result, the performance analysis will need to be revisited if more realistic models for fluid antenna become available in the future. Note that a brief discussion on how the impact of atmospheric conditions on fluid antenna could be minimized was given in [36].

Another limitation is that $5 \mathrm{G}$ and certainly next generations will be moving towards higher frequency bands. Consequently, the channel will have less multipath and be more directional [42]. In this case, it will be beneficial to accommodate more users to cause 'more' fading although this will require a large number of ports for the fluid antenna at each MS user. On the same spirit, it can be an exciting opportunity to combine the emerging intelligent reflecting surface (IRS) technology [43], [44] with fluid antenna, as IRS can provide a huge number of artificial scatterers for fading to occur for the exploitation of FAMA. Such idea is discussed in a recent paper [45].

Last but not least, FAMA only works if a sufficient number of ports is available at each user so that the interference fades deeply in at least one of those ports for multiple access. This begs the question if it is physically possible to install so many ports or how many ports would be realistic in practice. While this is indeed a growing research topic on its own, it is worth pointing out that the implementation in [46] allows seemingly an infinite resolution of ports for the fluid antenna design. In this design, radiation emits when a surface wave propagates on a substrate to cause a radio diffraction from the fluid radiator whose position can be changed using a micro-pump system. This effectively eliminates the need to install physical ports at different locations and yet provides the phenomenon of taking the radiation pattern of the fluid radiator at a different location. The number of realizable ports is therefore limited only by the control resolution of the micro-pump. Hence, the settings considered in this paper are practically feasible.

\section{CONCLUSION}

This paper proposed a novel concept for multiple access, referred to as FAMA, which handles inter-user interference purely by scanning through the fading envelopes and picking the best over a number of ports closely located within a small linear space. FAMA is inspired by the intuition that all signals 

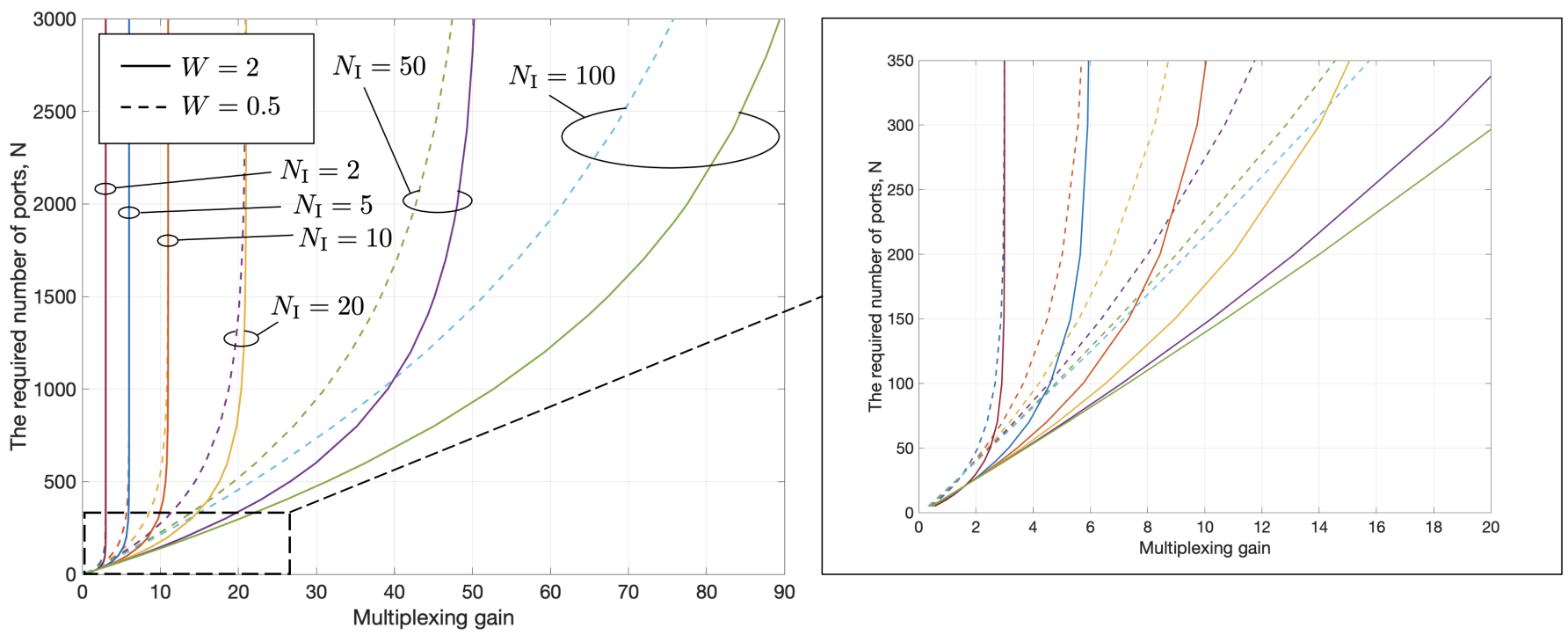

Fig. 7. The required number of ports, $N$, versus the multiplexing gain lower bound, $\underline{m}$, in (24) when $\gamma=10 \mathrm{~dB}$.

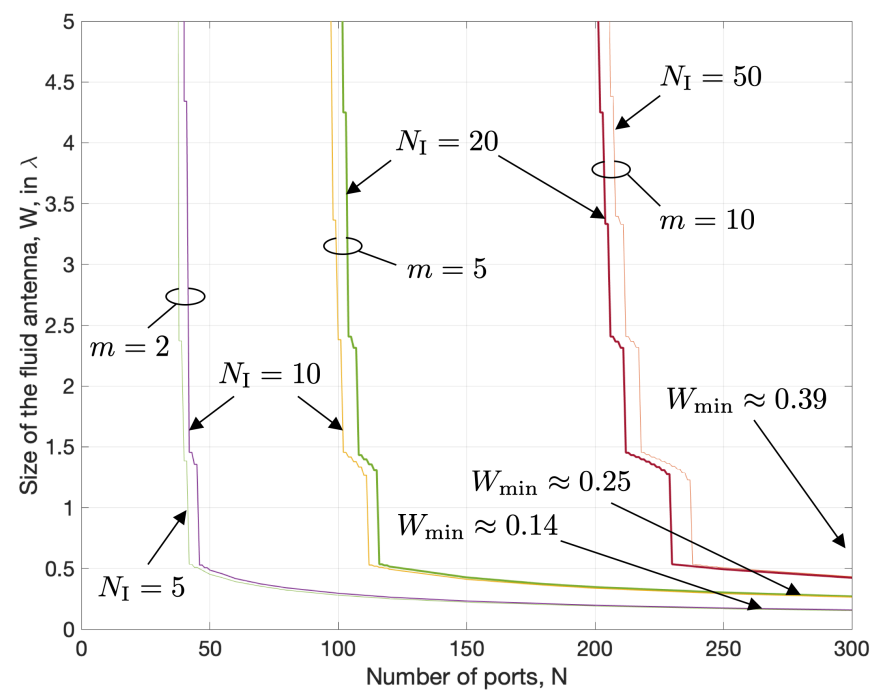

Fig. 8. The required size of fluid antenna, $W$, in (31) versus the number of ports when $\gamma=10 \mathrm{~dB}$.

including the interference suffer from deep fades, and multiple access is possible if the moments of deep fades (in space) for interference are exploited. The concept is also motivated by the emerging fluid antenna technology for software-controlled position-flexible antennas. Theoretically, it was revealed that if the number of ports is sufficiently large, then a fluid antenna can achieve any arbitrarily small SIR outage probability, thus illustrating its feasibility for interference elimination. Besides, we analyzed the average outage capacity and the multiplexing gain of the FAMA network. In particular, we showed that the network multiplexing gain grows linearly with the number of ports at each user while it is ultimately limited by the number of users. Our results revealed that the size of the fluid antenna has most impact if it is smaller than $\frac{\lambda}{2}$ but its impact has a diminishing return in the network capacity if it grows beyond $\frac{\lambda}{2}$. Additionally, our results demonstrated that accommodating hundreds of users in the same radio resource is possible, all by a single fluid-antenna with a small size at each user in FAMA, thereby achieving a significant capacity gain.

\section{APPENDICES}

\section{A. Proof of Theorem 1}

By definition, we have (36) (see top of next page). The conditional cumulative distribution function (cdf),

$$
\operatorname{Prob}\left(\left|g_{1}\right|<\sqrt{\gamma} t_{1}, \ldots,\left|g_{N}\right|<\sqrt{\gamma} t_{N} \mid t_{1}, \ldots, t_{N}\right),
$$

is found by [36, Theorem 2] while the pdf, $p_{\left|g_{1}^{\mathrm{I}}\right|, \ldots,\left|g_{N}^{\mathrm{I}}\right|}(\ldots)$, is given by [36, Theorem 1]. As such, we obtain (38) (see next page). To evaluate the integration over $t_{k}$, we first note that

$$
\int_{t_{k}=0}^{\infty} \frac{2 t_{k}}{\sigma_{\mathrm{I}}^{2}\left(1-\mu_{k}^{2}\right)} e^{-\frac{t_{k}^{2}+\mu_{k}^{2} t_{1}^{2}}{\sigma_{\mathrm{I}}^{2}\left(1-\mu_{k}^{2}\right)}} I_{0}\left(\frac{2 \mu_{k} t_{1} t_{k}}{\sigma_{\mathrm{I}}^{2}\left(1-\mu_{k}^{2}\right)}\right) d t_{k}=1
$$

because this is the total probability for a Rician random variable. Then the following lemma is useful.

Lemma 1: The following identity is true:

$$
\begin{aligned}
\int_{0}^{\infty} x e^{-\frac{x^{2}}{2}} & I_{0}(c x) Q_{1}(b, a x) d x \\
=e^{\frac{c^{2}}{2}} & Q_{1}\left(\frac{b}{\sqrt{a^{2}+1}}, \frac{a c}{\sqrt{a^{2}+1}}\right) \\
& -\frac{a^{2}}{a^{2}+1} e^{\frac{c^{2}-b^{2}}{2\left(a^{2}+1\right)}} I_{0}\left(\frac{a b c}{a^{2}+1}\right) .
\end{aligned}
$$

Proof: This is the result in [40, (B.32)] when $p=1$.

Considering the other term under the integration of $t_{k}$ and changing the variable by $x=\frac{t_{k}}{\sqrt{\frac{\sigma_{1}^{2}}{2}\left(1-\mu_{k}^{2}\right)}}$, we get (41) and then using the result in Lemma 1 on the right hand side, it yields (42) (for both equations, see next page). Using (39) and (42) into (38), and setting $z=\frac{t_{1}^{2}}{\sigma_{\mathrm{I}}^{2}}$ give the final result. 


$$
\begin{aligned}
\operatorname{Prob}(\operatorname{SIR}<\bar{\gamma}) & =\operatorname{Prob}\left(\max _{k}\left\{\frac{\left|g_{k}\right|^{2}}{\left|g_{k}^{\mathrm{I}}\right|^{2}}\right\}<\gamma\right)=\operatorname{Prob}\left(\frac{\left|g_{1}\right|}{\left|g_{1}^{\mathrm{I}}\right|}<\sqrt{\gamma}, \frac{\left|g_{2}\right|}{\left|g_{2}^{\mathrm{I}}\right|}<\sqrt{\gamma}, \ldots, \frac{\left|g_{N}\right|}{\left|g_{N}^{\mathrm{I}}\right|}<\sqrt{\gamma}\right) \\
& =\int_{0}^{\infty} \cdots \int_{0}^{\infty} \operatorname{Prob}\left(\left|g_{1}\right|<\sqrt{\gamma} t_{1}, \ldots,\left|g_{N}\right|<\sqrt{\gamma} t_{N} \mid t_{1}, \ldots, t_{N}\right) p_{\left|g_{1}^{\mathrm{I}}\right|, \ldots,\left|g_{N}^{\mathrm{I}}\right|}\left(t_{1}, \ldots, t_{N}\right) d t_{1} \cdots d t_{N}
\end{aligned}
$$

$$
\begin{aligned}
& \operatorname{Prob}(\operatorname{SIR}<\bar{\gamma})=\int_{t_{1}=0}^{\infty} \frac{2 t_{1}}{\sigma_{\mathrm{I}}^{2}} e^{-\frac{t_{1}^{2}}{\sigma_{\mathrm{I}}^{2}}} \int_{t=0}^{\frac{\gamma t_{1}^{2}}{\sigma^{2}}} e^{-t} \prod_{k=2}^{N}\{ \\
& \left.\int_{t_{k}=0}^{\infty}\left[1-Q_{1}\left(\sqrt{\frac{2 \mu_{k}^{2}}{1-\mu_{k}^{2}}} \sqrt{t}, \sqrt{\frac{2}{\sigma^{2}\left(1-\mu_{k}^{2}\right)}} \sqrt{\gamma} t_{k}\right)\right] \frac{2 t_{k}}{\sigma_{\mathrm{I}}^{2}\left(1-\mu_{k}^{2}\right)} e^{-\frac{t_{k}^{2}+\mu_{k}^{2} t_{1}^{2}}{\sigma_{\mathrm{I}}^{2}\left(1-\mu_{k}^{2}\right)}} I_{0}\left(\frac{2 \mu_{k} t_{1} t_{k}}{\sigma_{\mathrm{I}}^{2}\left(1-\mu_{k}^{2}\right)}\right) d t_{k}\right\} d t d t_{1}
\end{aligned}
$$

$$
\begin{aligned}
\int_{0}^{\infty} Q_{1}\left(\sqrt{\frac{2 \mu_{k}^{2}}{1-\mu_{k}^{2}}} \sqrt{t}, \sqrt{\frac{2}{\sigma^{2}\left(1-\mu_{k}^{2}\right)}} \sqrt{\gamma} t_{k}\right) \frac{2 t_{k}}{\sigma_{\mathrm{I}}^{2}\left(1-\mu_{k}^{2}\right)} e^{-\frac{t_{k}^{2}+\mu_{k}^{2} t_{1}^{2}}{\sigma_{\mathrm{I}}^{2}\left(1-\mu_{k}^{2}\right)}} I_{0}\left(\frac{2 \mu_{k} t_{1} t_{k}}{\sigma_{\mathrm{I}}^{2}\left(1-\mu_{k}^{2}\right)}\right) d t_{k} \\
=e^{-\frac{\mu_{k}^{2} t_{1}^{2}}{\sigma_{\mathrm{I}}^{2}\left(1-\mu_{k}^{2}\right)}} \int_{0}^{\infty} x e^{-\frac{x^{2}}{2}} I_{0}\left(\frac{\mu_{k} t_{1}}{\sqrt{\frac{\sigma_{\mathrm{I}}^{2}}{2}\left(1-\mu_{k}^{2}\right)}} x\right) Q_{1}\left(\sqrt{\left.\frac{2 \mu_{k}^{2}}{1-\mu_{k}^{2}} \sqrt{t}, \frac{\sigma_{\mathrm{I}} \sqrt{\gamma}}{\sigma} x\right) d x}\right.
\end{aligned}
$$

$$
\begin{aligned}
\int_{0}^{\infty} Q_{1}\left(\sqrt{\frac{2 \mu_{k}^{2}}{1-\mu_{k}^{2}}} \sqrt{t}, \sqrt{\frac{2}{\sigma^{2}\left(1-\mu_{k}^{2}\right)}} \sqrt{\gamma} t_{k}\right) \frac{2 t_{k}}{\sigma_{\mathrm{I}}^{2}\left(1-\mu_{k}^{2}\right)} e^{-\frac{t_{k}^{2}+\mu_{k}^{2} t_{1}^{2}}{\sigma_{\mathrm{I}}^{2}\left(1-\mu_{k}^{2}\right)}} I_{0}\left(\frac{2 \mu_{k} t_{1} t_{k}}{\sigma_{\mathrm{I}}^{2}\left(1-\mu_{k}^{2}\right)}\right) d t_{k} \\
=Q_{1}\left(\frac{1}{\sqrt{\frac{\sigma_{\mathrm{I}}^{2} \gamma}{\sigma^{2}}+1}} \sqrt{\frac{2 \mu_{k}^{2}}{1-\mu_{k}^{2}}} \sqrt{t}, \sqrt{\left.\frac{\frac{\sigma_{\mathrm{I}}^{2} \gamma}{\sigma^{2}}}{\frac{\sigma_{\mathrm{I}}^{2} \gamma}{\sigma^{2}}+1} \sqrt{\frac{2 \mu_{k}^{2}}{1-\mu_{k}^{2}}} t_{1}\right)}\right. \\
-\left(\frac{\frac{\sigma_{\mathrm{I}}^{2} \gamma}{\sigma^{2}}}{\frac{\sigma_{\mathrm{I}}^{2} \gamma}{\sigma^{2}}+1}\right) e^{-\left(\frac{1}{\frac{\sigma_{\mathrm{I}}^{2} \gamma}{\sigma^{2}}+1}\right) \frac{\mu_{k}^{2}}{1-\mu_{k}^{2}}\left(\frac{\gamma t_{1}^{2}}{\sigma^{2}}+t\right)} I_{0}\left(\frac{\frac{\sigma_{\mathrm{I}} \sqrt{\gamma}}{\sigma}}{\frac{\sigma_{\mathrm{I}}^{2} \gamma}{\sigma^{2}}+1}\left(\frac{2 \mu_{k}^{2}}{\sigma_{\mathrm{I}}\left(1-\mu_{k}^{2}\right)}\right) t_{1} \sqrt{t}\right)
\end{aligned}
$$

\section{B. Proof of Theorem 2}

To start with, we need the following lemma.

Lemma 2: We have the following lower bound:

$$
Q_{1}(\alpha, \beta) \geq e^{-\frac{\alpha^{2}+\beta^{2}}{2}} I_{0}(\alpha \beta) \text {. }
$$

Proof: This can be obtained by taking only the first term of the definition in [40, (A.5)].

Using Lemma 2 on the $Q_{1}(\cdot, \cdot)$ term inside the integration of (17) gives

$$
\begin{gathered}
Q_{1}\left(\frac{1}{\sqrt{\frac{\sigma_{1}^{2} \gamma}{\sigma^{2}}+1}} \sqrt{\frac{2 \mu_{k}^{2}}{1-\mu_{k}^{2}}} \sqrt{t}, \sqrt{\frac{\frac{\sigma_{1}^{2} \gamma}{\sigma^{2}}}{\frac{\sigma_{I}^{2} \gamma}{\sigma^{2}}+1}} \sqrt{\frac{2 \mu_{k}^{2}}{1-\mu_{k}^{2}}} \sqrt{z}\right) \\
\geq e^{-\left(\frac{1}{\frac{\sigma_{1}^{2} \gamma}{\sigma^{2}}+1}\right) \frac{\mu_{k}^{2}}{1-\mu_{k}^{2}}\left(\frac{\sigma_{1}^{2} \gamma}{\sigma^{2}} z+t\right)} \times \\
I_{0}\left(\frac{\frac{\sigma_{\mathrm{I}} \sqrt{\gamma}}{\sigma}}{\frac{\sigma_{I}^{2} \gamma}{\sigma^{2}}+1}\left(\frac{2 \mu_{k}^{2}}{1-\mu_{k}^{2}}\right) \sqrt{z t}\right) .
\end{gathered}
$$

Then apply the above result and evaluate the difference (45) where $(a)$ is from Lemma 2, $(c)$ uses the lower bound $I_{0}(x) \geq$ $\frac{e^{x}}{1+2 x}$ in $[41,(3.1)]$, and $(d)$ substitutes $t=0$ in the numerator of $(c)$ and $t=\frac{\sigma_{\mathrm{I}}^{2} \gamma}{\sigma^{2}} z$ in the denominator of $(c)$. Now, using (45) in the integration over $t$ in the outage probability (17) and performing a simple integration gives the final upper bound.

\section{Proof of Theorem 3}

Using the condition $\left|\mu_{2}\right|=\cdots=\left|\mu_{N}\right|=\mu$ and noting that $1-e^{-\frac{\sigma_{1}^{2} \gamma}{\sigma^{2}} z} \leq 1$, we have (46). Then after applying binomial expansion, we obtain (47) (see next page for both expressions). Using the fact that $\int_{0}^{\infty}\left(\frac{e^{-a x}}{1+b x}\right)^{k} d x=\frac{e^{\frac{k a}{b}}}{b} E_{k}\left(\frac{k a}{b}\right)$ in (47) and assuming $\frac{\sigma_{I}^{2} \gamma}{\sigma^{2}} \gg 1$ for scenarios with large interference and an ambitious SIR target give the desired result.

\section{Extension to Non-Homogeneous User Cases}

In general, each user may suffer from different interference power, $\sigma_{\mathrm{I}}^{2}$, and have different channel power, $\sigma^{2}$. As such, the achievable SIR outage probability will be different for each user, and each user will have a different average outage rate

$$
C_{u}=\left(1-p_{u}\right) \log _{2}(1+\bar{\gamma})
$$




$$
\begin{aligned}
& \left(\frac{\frac{\sigma_{\mathrm{I}}^{2} \gamma}{\sigma^{2}}}{\frac{\sigma_{\mathrm{I}}^{2} \gamma}{\sigma^{2}}+1}\right) e^{-\left(\frac{1}{\frac{\sigma_{1}^{2} \gamma}{\sigma^{2}}+1}\right) \frac{\mu_{k}^{2}}{1-\mu_{k}^{2}}\left(\frac{\sigma_{\mathrm{I}}^{2} \gamma}{\sigma^{2}} z+t\right)} I_{0}\left(\frac{\frac{\sigma_{\mathrm{I}} \sqrt{\gamma}}{\sigma}}{\frac{\sigma_{\mathrm{I}}^{2} \gamma}{\sigma^{2}}+1}\left(\frac{2 \mu_{k}^{2}}{1-\mu_{k}^{2}}\right) \sqrt{z t}\right) \\
& -Q_{1}\left(\frac{1}{\sqrt{\frac{\sigma_{I}^{2} \gamma}{\sigma^{2}}+1}} \sqrt{\frac{2 \mu_{k}^{2}}{1-\mu_{k}^{2}}} \sqrt{t}, \sqrt{\frac{\frac{\sigma_{I}^{2} \gamma}{\sigma^{2}}}{\frac{\sigma_{I}^{2} \gamma}{\sigma^{2}}+1}} \sqrt{\frac{2 \mu_{k}^{2}}{1-\mu_{k}^{2}}} \sqrt{z}\right) \\
& \stackrel{(a)}{\leq}\left(\frac{\frac{\sigma_{\mathrm{I}}^{2} \gamma}{\sigma^{2}}}{\frac{\sigma_{\mathrm{I}}^{2} \gamma}{\sigma^{2}}+1}\right) e^{-\left(\frac{1}{\frac{\sigma_{1}^{2} \gamma}{\sigma^{2}}+1}\right) \frac{\mu_{k}^{2}}{1-\mu_{k}^{2}}\left(\frac{\sigma_{\mathrm{I}}^{2} \gamma}{\sigma^{2}} z+t\right)} I_{0}\left(\frac{\frac{\sigma_{\mathrm{I}} \sqrt{\gamma}}{\sigma}}{\frac{\sigma_{\mathrm{I}}^{2} \gamma}{\sigma^{2}}+1}\left(\frac{2 \mu_{k}^{2}}{1-\mu_{k}^{2}}\right) \sqrt{z t}\right) \\
& -e^{-\left(\frac{1}{\frac{\sigma_{I}^{2} \gamma}{\sigma^{2}}+1}\right) \frac{\mu_{k}^{2}}{1-\mu_{k}^{2}}\left(\frac{\sigma_{I}^{2} \gamma}{\sigma^{2}} z+t\right)} I_{0}\left(\frac{\frac{\sigma_{\mathrm{I}} \sqrt{\gamma}}{\sigma}}{\frac{\sigma_{I}^{2} \gamma}{\sigma^{2}}+1}\left(\frac{2 \mu_{k}^{2}}{1-\mu_{k}^{2}}\right) \sqrt{z t}\right) \\
& \stackrel{(b)}{=}\left(\frac{-1}{\frac{\sigma_{I}^{2} \gamma}{\sigma^{2}}+1}\right) e^{-\left(\frac{1}{\frac{\sigma_{I}^{2} \gamma}{\sigma^{2}}+1}\right) \frac{\mu_{k}^{2}}{1-\mu_{k}^{2}}\left(\frac{\sigma_{I}^{2} \gamma}{\sigma^{2}} z+t\right)} I_{0}\left(\frac{\frac{\sigma_{\mathrm{I}} \sqrt{\gamma}}{\sigma}}{\frac{\sigma_{\mathrm{I}}^{2} \gamma}{\sigma^{2}}+1}\left(\frac{2 \mu_{k}^{2}}{1-\mu_{k}^{2}}\right) \sqrt{z t}\right) \\
& \stackrel{(c)}{\leq}\left(\frac{-1}{\frac{\sigma_{1}^{2} \gamma}{\sigma^{2}}+1}\right) \frac{e^{-\left(\frac{1}{\frac{\sigma_{1}^{2} \gamma}{\sigma^{2}}+1}\right) \frac{\mu_{k}^{2}}{1-\mu_{k}^{2}}\left(\frac{\sigma_{\mathrm{I}} \sqrt{\gamma}}{\sigma} \sqrt{z}-\sqrt{t}\right)^{2}}}{1+4\left(\frac{1}{\frac{\sigma_{1}^{2} \gamma}{\sigma^{2}}+1}\right)\left(\frac{\mu_{k}^{2}}{1-\mu_{k}^{2}}\right) \frac{\sigma_{\mathrm{I}} \sqrt{\gamma}}{\sigma} \sqrt{z t}} \stackrel{(d)}{\leq}\left(\frac{-1}{\frac{\sigma_{I}^{2} \gamma}{\sigma^{2}}+1}\right) \frac{e^{-\left(\frac{1}{\frac{\sigma_{1}^{2} \gamma}{\sigma^{2}}+1}\right)\left(\frac{\mu_{k}^{2}}{1-\mu_{k}^{2}}\right) \frac{\sigma_{1}^{2} \gamma}{\sigma^{2}} z}}{1+4\left(\frac{1}{\frac{\sigma_{I}^{2} \gamma}{\sigma^{2}}+1}\right)\left(\frac{\mu_{k}^{2}}{1-\mu_{k}^{2}}\right) \frac{\sigma_{1}^{2} \gamma}{\sigma^{2}} z},
\end{aligned}
$$

$$
\operatorname{Prob}(\mathrm{SIR}<\gamma) \leq \varepsilon_{\mathrm{UB}}^{\mathrm{I}} \leq \varepsilon_{\mathrm{UB}}^{\mathrm{II}}=\int_{0}^{\infty} e^{-z}\left[1-\left(\frac{1}{\frac{\sigma_{\mathrm{I}}^{2} \gamma}{\sigma^{2}}+1}\right) \frac{e^{-\left(\frac{1}{\frac{\sigma_{1}^{2} \gamma}{\sigma^{2}}+1}\right)\left(\frac{\mu^{2}}{1-\mu^{2}}\right) \frac{\sigma_{\mathrm{I}}^{2} \gamma}{\sigma^{2}} z}}{1+4\left(\frac{1}{\frac{\sigma_{\mathrm{I}}^{2} \gamma}{\sigma^{2}}+1}\right)\left(\frac{\mu^{2}}{1-\mu^{2}}\right) \frac{\sigma_{\mathrm{I}}^{2} \gamma}{\sigma^{2}} z}\right]^{N-1} d z
$$

$$
\begin{aligned}
\varepsilon_{\mathrm{UB}}^{\mathrm{II}} & =\int_{0}^{\infty} e^{-z} \sum_{k=0}^{N-1}\left(\begin{array}{c}
N-1 \\
k
\end{array}\right) \frac{(-1)^{k}}{\left(\frac{\sigma_{\mathrm{I}}^{2} \gamma}{\sigma^{2}}+1\right)^{k}}\left[\frac{e^{-\left(\frac{1}{\frac{\sigma_{\mathrm{I}}^{2} \gamma}{\sigma^{2}}+1}\right)\left(\frac{\mu^{2}}{1-\mu^{2}}\right) \frac{\sigma_{\mathrm{I}}^{2} \gamma}{\sigma^{2}} z}}{1+4\left(\frac{1}{\frac{\sigma_{\mathrm{I}}^{2} \gamma}{\sigma^{2}}+1}\right)\left(\frac{\mu^{2}}{1-\mu^{2}}\right) \frac{\sigma_{\mathrm{I}}^{2} \gamma}{\sigma^{2}} z}\right]^{k} d z \\
& =\sum_{k=0}^{N-1} \frac{\left(\begin{array}{c}
N-1 \\
k
\end{array}\right)(-1)^{k}}{\left(\frac{\sigma_{\mathrm{I}}^{2} \gamma}{\sigma^{2}}+1\right)^{k}} \int_{0}^{\infty}\left\{\frac{\left.e^{-\left[\left(\frac{1}{\sigma_{\mathrm{I}}^{2} \gamma}\right)\left(\frac{\mu^{2}}{\sigma^{2}}+1\right)\right.} \frac{\sigma_{\mathrm{I}}^{2} \gamma}{\sigma^{2}}+\frac{1}{k}\right] z}{1+4\left(\frac{1}{\frac{\sigma_{\mathrm{I}}^{2} \gamma}{\sigma^{2}}+1}\right)\left(\frac{\mu^{2}}{1-\mu^{2}}\right) \frac{\sigma_{\mathrm{I}}^{2} \gamma}{\sigma^{2}} z}\right\}^{k} d z
\end{aligned}
$$

where $p_{u}$ is the outage probability for user $u$ (computed using Theorem 1 with appropriate values of $\sigma_{\mathrm{I}}^{2}$ and $\sigma^{2}$ ). The overall average network outage rate therefore is given by

$$
C_{\text {FAMA }}(\gamma)=\sum_{u=1}^{N_{\mathrm{I}}+1} C_{u}=\sum_{u=1}^{N_{\mathrm{I}}+1}\left(1-p_{u}\right) \ln (1+\bar{\gamma}) .
$$

As a result, the multiplexing gain then takes the general form

$$
\begin{aligned}
m=\frac{C_{\text {FAMA }}(\gamma)}{C_{\text {single-user }}(\gamma)} & =\frac{\sum_{u=1}^{N_{\mathrm{I}}+1}\left(1-p_{u}\right) \log _{2}(1+\bar{\gamma})}{\log _{2}(1+\bar{\gamma})} \\
& =\sum_{u=1}^{N_{\mathrm{I}}+1}\left(1-p_{u}\right) .
\end{aligned}
$$

If all the users have the same outage probability $p$, then (50) is reduced to $m=\left(N_{\mathrm{I}}+1\right)(1-p)$.

Note that in the literature, the following definition of mul- 
tiplexing gain is usually used

$$
m=\lim _{\bar{\gamma} \rightarrow \infty} \frac{C_{\text {network }}}{\ln \bar{\gamma}}
$$

which measures how the network capacity, $C_{\text {network}}$, scales against the SNR (or SINR) in the log scale. The expectation is that if $C_{\text {network }}$ is in the form $m \ln (1+\bar{\gamma}),(51)$ will return the capacity scaling factor $m$ over a single-user system for large $\bar{\gamma}$. The definition in (51) would be needed if the transmitter adapts its coding rate to match the ergodic capacity of the channel. In this case, $\bar{\gamma}$ would be a random variable depending on the channel quality. By contrast, in this paper, all the transmitters have a fixed coding rate, so (50) is more appropriate.

\section{E. Proof of Theorem 5}

The result (25) comes directly from (24) when substituting (20). Now, if $\mu$ is reasonably small, then $\frac{k}{4}+\frac{1-\mu^{2}}{4 \mu^{2}}$ will be large. As such, we can approximate $E_{k}(x) \approx \frac{e^{-x}}{x}$. We can therefore rewrite (25) as

$$
\begin{aligned}
& 1+\sum_{k=1}^{N-1} \frac{\left(\begin{array}{c}
N-1 \\
k
\end{array}\right)(-1)^{k}}{\left(\frac{\sigma_{\mathrm{I}}^{2} \gamma}{\sigma^{2}}\right)^{k}}\left(\frac{1-\mu^{2}}{(k-1) \mu^{2}+1}\right) \leq 1-\frac{m}{N_{\mathrm{I}}+1}, \\
\Leftrightarrow & \sum_{k=1}^{N-1} \frac{\left(\begin{array}{c}
N-1 \\
k
\end{array}\right)(-1)^{k+1}}{\left(\frac{\sigma_{\mathrm{I}}^{2} \gamma}{\sigma^{2}}\right)^{k}}\left(\frac{1-\mu^{2}}{1+(k-1) \mu^{2}}\right) \geq \frac{m}{N_{\mathrm{I}}+1} .
\end{aligned}
$$

Noting that we have the Taylor series

$$
\begin{aligned}
\frac{1-\mu^{2}}{1+(k-1) \mu^{2}} & =1-k \mu^{2}+(k-1) k \mu^{4}-(k-1)^{2} k \mu^{6}+\cdots \\
& \approx 1-k \mu^{2}, \text { for small } \mu,
\end{aligned}
$$

we use this approximation into (52) to get (26). The condition (27) can also be easily obtained by keeping only the first term in the summation which is valid if $\frac{\sigma_{I}^{2} \gamma}{\sigma^{2}}$ is very large.

\section{F. Proof of Theorem 7}

To prove the result, we interpret the fluid antenna system as a linear space of $W \lambda$ that has $N$ ports having cross-correlation parameters in decreasing order, i.e.,

$$
\left|\mu_{2}\right| \geq\left|\mu_{3}\right| \geq \cdots \geq\left|\mu_{N}\right|,
$$

as the position of the port moves away from the reference position, i.e., port 1 . The property in (54) does not normally apply because Bessel function $J_{0}(\cdot)$ is an oscillating function as the distance increases. However, as the distance from the reference position increases, the general trend of $\left|J_{0}(\cdot)\right|$ does go down. By defining the inverse of $J_{0}(\cdot)$ as the function $\rho^{*}=J_{0}^{-1}\left(\mu^{*}\right)$ that always returns the minimum value of $\rho^{*}$ to ensure $\left|J_{0}(\rho)\right| \leq \mu^{*}$ for $\rho \geq \rho^{*}$ and enforce the monotonicity, we can construct a fluid antenna model where the ports farther away contribute more diversity (and so less cross-correlation) than the near ones (which agrees with the intuition).

Based on this model, the performance of an $N$-port fluid antenna system is lower bounded by that of the same system, keeping only ports $\frac{N}{2}+1, \ldots, N$ but dropping the ports from 1 to $\left\lfloor\frac{N}{2}\right\rfloor$. This performance is further lower bounded by setting the cross-correlation parameters as the one at $\frac{W \lambda}{2}$, i.e.,

$$
\begin{aligned}
\left|\mu_{\left\lfloor\frac{N}{2}\right\rfloor+1}\right|=\left|\mu_{\left\lfloor\frac{N}{2}\right\rfloor+2}\right|=\cdots=\left|\mu_{N}\right| & =\left|J_{0}\left(\frac{2 \pi}{\lambda} \frac{W \lambda}{2}\right)\right| \\
& =\left|J_{0}(\pi W)\right| .
\end{aligned}
$$

Setting $\left|J_{0}(\pi W)\right|=\mu^{*}$ in (30) with these $\left\lfloor\frac{N}{2}\right\rfloor$ ports, and using $J_{0}^{-1}\left(\mu^{*}\right)$ provide a sufficient condition for $W$ to achieve the SIR target and multiplexing gain.

\section{REFERENCES}

[1] A. J. Paulraj, and T. Kailath, "Increasing capacity in wireless broadcast systems using distributed transmission/directional reception (DTDR)," US Patent 5,345,599A, granted 1994.

[2] G. J. Foschini, and M. J. Gans, "On limits of wireless communications in a fading environment when using multiple antennas," Wireless Pers. Commun., vol. 6, no. 3, pp. 311-335, Mar. 1998.

[3] V. Tarokh, N. Seshadri, and A. R. Calderbank, "Space-time codes for high data rate wireless communication: Performance criterion and code construction," IEEE Trans. Inform. Theory, vol. 44, no. 2, pp. 744-765, Mar. 1998.

[4] S. M. Alamouti, "A simple transmit diversity technique for wireless communications," IEEE J. Select. Areas Commun., vol. 16, no. 8, pp. 1451-1458, Oct. 1998.

[5] L. Zheng, and D. N. C. Tse, "Diversity and multiplexing: A fundamental tradeoff in multiple-antenna channels," IEEE Trans. Inform. Theory, vol. 49, no. 5, pp. 1073-1096, May 2003.

[6] S. Vishwanath, N. Jindal, and A. Goldsmith, "Duality, achievable rates, and sum-rate capacity of Gaussian MIMO broadcast channels," IEEE Trans. Inform. Theory, vol. 49, no. 10, pp. 2658-2668, Oct. 2003.

[7] Q. H. Spencer, A. L. Swindlehurst, and M. Haardt, "Zero-forcing methods for downlink spatial multiplexing in multiuser MIMO channels," IEEE Trans. Signal Proc., vol. 52, no. 2, pp. 461-471, Feb. 2004.

[8] H. Q. Ngo, E. G. Larsson, and T. L. Marzetta, "Energy and spectral efficiency of very large multiuser MIMO systems," IEEE Trans. Commun., vol. 61, no. 4, pp. 1436-1449, Apr. 2013.

[9] E. G. Larsson, O. Edfors, F. Tufvesson, and T. L. Marzetta, "Massive MIMO for next generation wireless systems," IEEE Commun. Mag., vol. 52, no. 2, pp. 186-195, Feb. 2014.

[10] P. von Butovitsch, D. Astely, C. Friberg, A. Furuskär, B. Göransson, B. Hogan, J. Karlsson, and E. Larsson, "Advanced antenna systems for 5G networks," [Online] https://www.ericsson.com/en/white-papers/ advanced-antenna-systems-for-5g-networks, White paper, Accessed: 2019-08-07.

[11] "Sprint unveils six 5G-ready cities: Significant milestone toward launching first 5G mobile network in the U.S.," [Online] https://newsroom.sprint. com/sprint-unveils-5g-ready-massive-mimo-markets.htm, Accessed: 2019-08-07.

[12] "Huawei launches 5G simplified solution," [Online] https://www. huawei.com/en/press-events/news/2019/2/huawei-5g-simplified-solution, Feb. 2019.

[13] H. Yin, L. Cottatellucci, D. Gesbert, R. R. Müller, and G. He, "Robust pilot decontamination based on joint angle and power domain discrimination," IEEE Trans. Signal Process., vol. 64, no. 11, pp. 2990-3003, 2016.

[14] H. V. Cheng, E. Björnson, and E. G. Larsson, "Optimal pilot and payload power control in single-cell massive MIMO systems," IEEE Trans. Signal Process., vol. 65, no. 9, pp. 2363-2378, 2017.

[15] D. Bethanabhotla, O. Y. Bursalioglu, H. C. Papadopoulos, and G. Caire, "Optimal user-cell association for massive MIMO wireless networks," IEEE Trans. Wireless Commun., vol. 15, no. 3, pp. 1835-1850, Mar. 2016.

[16] M. Karimzadeh-Farshbafan, V. Shah-Mansouri, and D. Niyato, "A dynamic reliability-aware service placement for network function virtualization (NFV)," IEEE J. Select. Areas Commun., vol. 38, no. 22, pp. 318-333, Feb. 2020.

[17] Y. Jiang, Y. Hu, M. Bennis, F.-C. Zheng, and X. You, "A mean field game-based distributed edge caching in fog radio access networks," IEEE Trans. Commun., vol. 68, no. 3, pp. 1567-1580, Mar. 2020.

[18] K. K. Wong, K. F. Tong, Y. Zhang, and Z. Zheng, "Fluid antenna system for 6G: When Bruce Lee inspires wireless communications," IET Electronics Letters, vol. 56, no. 24, pp. 1288-1290, 26 Nov. 2020. 
[19] S. J. Kar, A. Chakrabarty and B. K. Sarkar, "Fluid antennas," in Proc. IEEE Middle East Conf. Antennas and Propag. (MECAP 2010), pp. 1-6, 20-22 Oct. 2010, Cairo, Egypt.

[20] Y. Kosta, and S. Kosta, "Liquid antenna systems," in Proc. IEEE Antennas and Propag. Society Symposium, vol. 3, pp. 2392-2395, 2025 Jun. 2004, Monterey, CA, USA.

[21] G. J. Hayes, J.-H. So, A. Qusba, M. D. Dickey, and G. Lazzi, "Flexible liquid metal alloy (EGaIn) microstrip patch antenna," IEEE Trans. Antennas Propag., vol. 60, no. 5, pp. 2151-2156, May 2012.

[22] A. M. Morishita, C. K. Y. Kitamura, A. T. Ohta, and W. A. Shiroma "A liquid-metal monopole array with tunable frequency, gain, and beam steering," IEEE Antennas Wireless Propag. Lett., vol. 12, pp. 1388-1391, 2013.

[23] A. Dey, R. Guldiken, and G. Mumcu, "Microfluidically reconfigured wideband frequency-tunable liquid-metal monopole antenna," IEEE Trans. Antennas Propag., vol. 64, no. 6, pp. 2572-2576, Jun. 2016.

[24] A. P. Saghati, J. Batra, J. Kameoka, and K. Entesari, "A microfluidicallytuned dual-band slot antenna," in Proc. IEEE Antennas Propag. Soc. Int. Symp. (APSURSI), pp. 1244-1245, 6-11 Jul. 2014, Memphis, TN, USA.

[25] C. Borda-Fortuny, K.-F. Tong, A. Al-Armaghany, and K. K. Wong, "A low-cost fluid switch for frequency-reconfigurable Vivaldi antenna," IEEE Antennas Wireless Propag. Lett., vol. 16, pp. 3151-3154, 2017.

[26] C. Borda-Fortuny, K. F. Tong, and K. Chetty, "Low-cost mechanism to reconfigure the operating frequency band of a Vivaldi antenna for cognitive radio and spectrum monitoring applications," IET Microwaves, Antennas \& Propag., vol. 12, no. 5, pp. 779-782, 2018.

[27] C. Borda-Fortuny, L. Cai, K. F. Tong, and K. K. Wong, "Low-cost 3Dprinted coupling-fed frequency agile fluidic monopole antenna system," IEEE Access, pp. 95058-95064, Jul. 2019.

[28] A. Singh, I. Goode, and C. E. Saavedra, "A multistate frequency reconfigurable monopole antenna using fluidic channels," IEEE Antennas Wireless Propag. Lett., vol. 18, no. 5, pp. 856-860, May 2019.

[29] S. Song, and R. D. Murch, "An efficient approach for optimizing frequency reconfigurable pixel antennas using genetic algorithms," IEEE Trans. Antennas Propag., vol. 62, no. 2, pp. 609-620, Feb. 2014.

[30] "Mitsubishi electric's SeaAerial antenna uses seawater plume," Available [online]: https://www.mitsubishielectric.com.

[31] L. Xing, J. Zhu, Q. Xu, D. Yan and Y. Zhao, "A circular beam-steering antenna with parasitic water reflectors," IEEE Antennas and Wireless Propag. Letters, vol. 18, no. 10, pp. 2140-2144, Oct. 2019.

[32] M. Hampson, "New antenna uses saltwater and plastic to steer radio beams," IEEE Spectrum-The Tech Talk blog, 2019.

[33] K. N. Paracha, A. D. Butt, A. S. Alghamdi, S. A. Babale, and P. J. Soh, "Liquid metal antennas: Materials, fabrication and applications," Sensors 2020, 20, 177.

[34] Y. Huang, L. Xing, C. Song, S. Wang and F. Elhouni, "Liquid antennas Past, present and future," IEEE Open J. Antennas and Propag., vol. 2, pp. 473-487, 2021.

[35] F. Tariq et al., "A speculative study on 6G," IEEE Wireless Commun., vol. 27 , no. 4, pp. 118-125, Aug. 2020.

[36] K. K. Wong, A. Shojaeifard, K.-F. Tong and Y. Zhang, "Fluid antenna systems," IEEE Trans. Wireless Commun., vol. 20, no. 3, pp. 1950-1962, Mar. 2021.

[37] K. K. Wong, A. Shojaeifard, K.-F. Tong and Y. Zhang, "Performance limits of fluid antenna systems," IEEE Commun. Letters, vol. 24, no. 11 pp. 2469-2472, Nov. 2020

[38] G. L. Stüber, Principles of Mobile Communication, Second Edition, Kluwer Academic Publishers, 2002.

[39] S. Choudhury and J. D. Gibson, "Information transmission over fading channels," in Proc. IEEE Global Telecomm. Conf., pp. 3316-3321, 26-30 Nov. 2007, Washington, DC, USA.

[40] M. K. Simon, Probability Distributions Involving Gaussian Random Variables: A Handbook for Engineers and Scientists, Springer, Boston, MA, 2002.

[41] Z.-H. Yang, and Y.-M. Chu, "On approximating the modified Bessel function of the first kind and Toader-Qi mean," J. Inequalities and Appl., vol. 40,2016

[42] I. A. Hemadeh, K. Satyanarayana, M. El-Hajjar and L. Hanzo, "Millimeter-wave communications: Physical channel models, design considerations, antenna constructions, and link-budget," IEEE Commun. Surveys \& Tut., vol. 20, no. 2, pp. 870-913, Secondquarter 2018.

[43] C. Liaskos et al., "A new wireless communication paradigm through software-controlled metasurfaces," IEEE Commun. Mag., vol. 56, no. 9 , pp. 162-169, Sept. 2018.

[44] E. Basar et al., "Wireless communications through reconfigurable intelligent surfaces," IEEE Access, vol. 7, pp. 116753-116773, 2019.
[45] A. Shojaeifard et al., "MIMO evolution beyond 5G through reconfigurable intelligent surfaces and fluid antenna systems," submitted.

[46] Y. Shen, K. F. Tong, and K. K. Wong, "Beam-steering surface wave fluid antennas for MIMO applications," in Proc. The 2020 Asia-Pacific Microwave Conf. (APMC 2020), 8-11 Dec. 2020, Hong Kong, China.

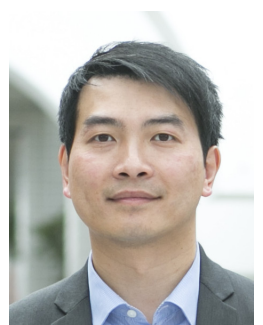

(Kit) Kai-Kit Wong (M'01-SM'08-F'16) received the BEng, the MPhil, and the PhD degrees, al in Electrical and Electronic Engineering, from the Hong Kong University of Science and Technology, Hong Kong, in 1996, 1998, and 2001, respectively. After graduation, he took up academic and research positions at the University of Hong Kong, Lucent Technologies, Bell-Labs, Holmdel, the Smart Antennas Research Group of Stanford University, and the University of Hull, UK. He is Chair in Wireless Communications at the Department of Electronic and Electrical Engineering, University College London, UK.

His current research centers around $5 \mathrm{G}$ and beyond mobile communications. $\mathrm{He}$ is a co-recipient of the 2013 IEEE Signal Processing Letters Best Paper Award and the 2000 IEEE VTS Japan Chapter Award at the IEEE Vehicular Technology Conference in Japan in 2000, and a few other international best paper awards. He is Fellow of IEEE and IET and is also on the editorial board of several international journals. He is the Editor-in-Chief for IEEE Wireless Communications Letters since 2020.

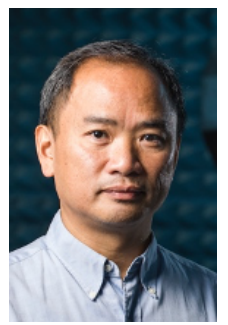

Kin Fai Tong (M'99-SM'13) received the B.Eng. and $\mathrm{Ph} . \mathrm{D}$. degrees in electronic engineering from the City University of Hong Kong in 1993 and 1997, respectively. After graduation, Dr. Tong worked in the Department of Electronic Engineering at City University of Hong Kong as a Research Fellow. Two years later, he took up the post Expert researcher in the Photonic Information Technology Group and Millimetre-wave Devices Group at the National Institute of Information and Communications Technology (NiCT), Japan, where his main research focused on photonic-millimeter-wave planar antennas at $10 \mathrm{GHz}$, $38 \mathrm{GHz}$ and $60 \mathrm{GHz}$ for high-speed wireless communications systems. In 2005, he started his academic career in the Department of Electronic and Electrical Engineering, UCL, as a lecturer. Now Dr. Tong is Chair in Antennas, Microwave and Millimeter-wave Engineering in the department. His current research interests include millimeter-wave and $\mathrm{THz}$ antennas, fluid antennas, 3D printed antennas and sub-GHz long range IoT networks. He served as the General Co-Chair of the 2017 International Workshop on Electromagnetics (iWEM), and Lead Guest Editor of IEEE OJAP in 2020. 